\title{
Donor dendritic cell-derived exosomes promote allograft-targeting immune response
}

\author{
Quan Liu,, ${ }^{1,2}$ Darling M. Rojas-Canales, ${ }^{1}$ Sherrie J. Divito, ${ }^{1}$ William J. Shufesky, ${ }^{1}$ Donna Beer Stolz, ${ }^{3}$ Geza Erdos, ${ }^{4}$ \\ Mara L.C. Sullivan, ${ }^{3}$ Gregory A. Gibson, ${ }^{3}$ Simon C. Watkins, ${ }^{3}$ Adriana T. Larregina, ${ }^{4,5,6}$ and Adrian E. Morellii, \\ TT.E. Starzl Transplantation Institute, Department of Surgery, University of Pittsburgh, Pittsburgh, Pennsylvania, USA. ²Department of Cardiovascular Surgery, The Second Affiliated Hospital \\ of Harbin Medical University, Harbin, Heilongjiang Province, China. ${ }^{3}$ Department of Cell Biology, ${ }^{4}$ Department of Dermatology, ${ }^{5}$ Department of Immunology, and ${ }^{6} \mathrm{McCowan}$ Institute \\ for Regenerative Medicine, University of Pittsburgh, Pittsburgh, Pennsylvania, USA.
}

\begin{abstract}
The immune response against transplanted allografts is one of the most potent reactions mounted by the immune system. The acute rejection response has been attributed to donor dendritic cells (DCs), which migrate to recipient lymphoid tissues and directly activate alloreactive T cells against donor MHC molecules. Here, using a murine heart transplant model, we determined that only a small number of donor DCs reach lymphoid tissues and investigated how this limited population of donor DCs efficiently initiates the alloreactive T cell response that causes acute rejection. In our mouse model, efficient passage of donor MHC molecules to recipient conventional DCs (cDCs) was dependent on the transfer of extracellular vesicles (EVs) from donor DCs that migrated from the graft to lymphoid tissues. These EVs shared characteristics with exosomes and were internalized or remained attached to the recipient cDCs. Recipient CDCs that acquired exosomes became activated and triggered full activation of alloreactive T cells. Depletion of recipient CDCs after cardiac transplantation drastically decreased presentation of donor MHC molecules to directly alloreactive T cells and delayed graft rejection in mice. These findings support a key role for transfer of donor EVs in the generation of allograft-targeting immune responses and suggest that interrupting this process has potential to dampen the immune response to allografts.
\end{abstract}

\section{Introduction}

In the absence of immunosuppression, the strong adaptive immune response against organ allografts is the main impediment to successful transplantation. The potency of the adaptive immune response to alloantigens on the graft is attributed to migration of donor-derived professional antigen-presenting cells (APCs) as dendritic cells (DCs) to the recipient lymphoid tissues, where the donor APCs trigger the activation of directly allospecific T cells against donor MHC molecules $(1,2)$. Nevertheless, the idea that donor leukocytes migrated from transplanted organs present by themselves intact MHC molecules to directly alloreactive $\mathrm{T}$ cells in situ in graft-draining lymphoid tissues has been questioned in recent years (3-7). There is indirect evidence in murine models that donor DCs mobilized from organ allografts home in recipient lymphoid tissues in relatively low numbers (2), are shortlived because they are targets for recipient NK cells and cytotoxic T lymphocytes (8-10), and do not interact efficiently with directly alloreactive T cells (11). Nevertheless, the donor DCs mobilized from mouse heart allografts to lymphoid tissues of naive recipients, even at what seems to be extremely low DC/T cell ratios, are able to elicit the potent antidonor response that acutely rejects the graft (2). By contrast, the allostimulatory ability of fully mature DCs is barely detectable in mixed leukocyte cultures below an APC/T cell ratio of 1:100 (12). These apparently contradictory findings have raised the question of how, in some transplanta-

Conflict of interest: The authors have declared that no conflict of interest exists. Submitted: September 10, 2015; Accepted: May 4, 2016.

Reference information: J Clin Invest. 2016;126(8):2805-2820. doi:10.1172/JCI84577. tion models, the limited number of graft-derived DCs that home in graft-draining lymphoid tissues activates so efficiently directly allospecific T cells. This is particularly intriguing in nonsensitized recipients, where naive $\mathrm{T}$ cells against alloantigens are present in relatively lower percentages and have a higher activation threshold than the allo- or cross-reactive memory $\mathrm{T}$ cells found in presensitized recipients.

Here, we demonstrated that the relatively few donor DCs mobilized from heart allografts to lymphoid tissues of naive recipients amplify their ability to stimulate directly alloreactive T cells by transferring clusters of extracellular vesicles (EVs), with characteristics of exosomes and bearing functional donor MHC molecules and APC-activating signals, to a higher number of recipient conventional DCs (cDCs). Exosomes are 70- to 120-nm EVs originated in the endocytic compartment of living cells, which have been shown to transfer proteins and RNAs between cells (13-16). We found that after cardiac transplantation, the donor-derived exosomes remain attached to or are internalized by recipient cDCs in graft-draining lymphoid tissues, but they did not fuse with the plasma membrane of the acceptor APCs. Uptake of donorderived exosomes, unlike interaction with other types of donor EVs, enhanced the ability of the acceptor (recipient) DCs to stimulate allospecific $\mathrm{T}$ cells. In accordance with the finding that recipient DCs present donor MHC molecules acquired through EVs to directly alloreactive $\mathrm{T}$ cells, depletion of recipient DCs abrogated activation of directly alloreactive $\mathrm{T}$ cells, and delayed allograft rejection. Our findings define a new role for exosome transfer, as a mechanism of spreading donor MHC molecules and APC-activating signals from a limited number of graft-derived migrating DCs 


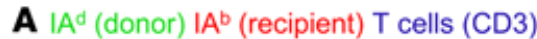
Cell gate (spleen): CD11 chivint
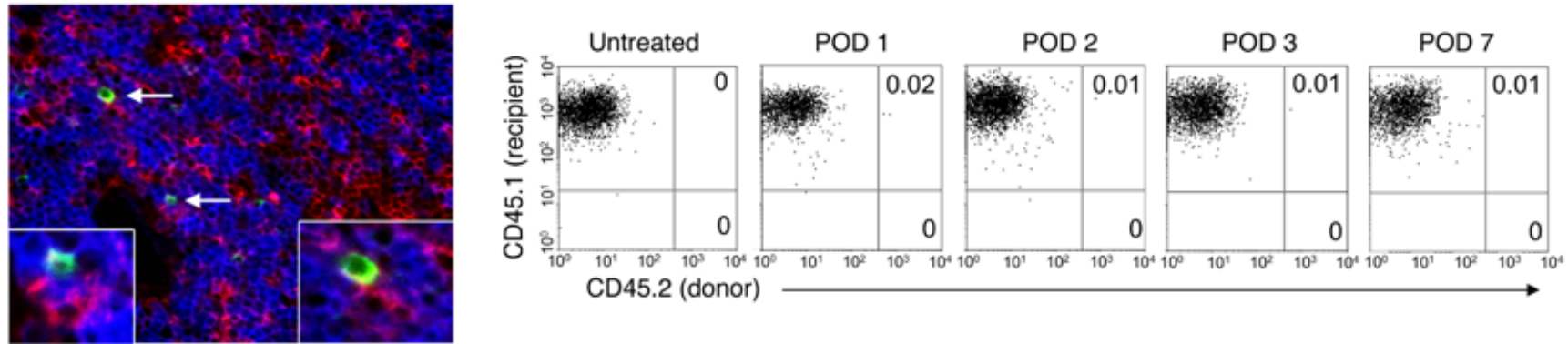

B
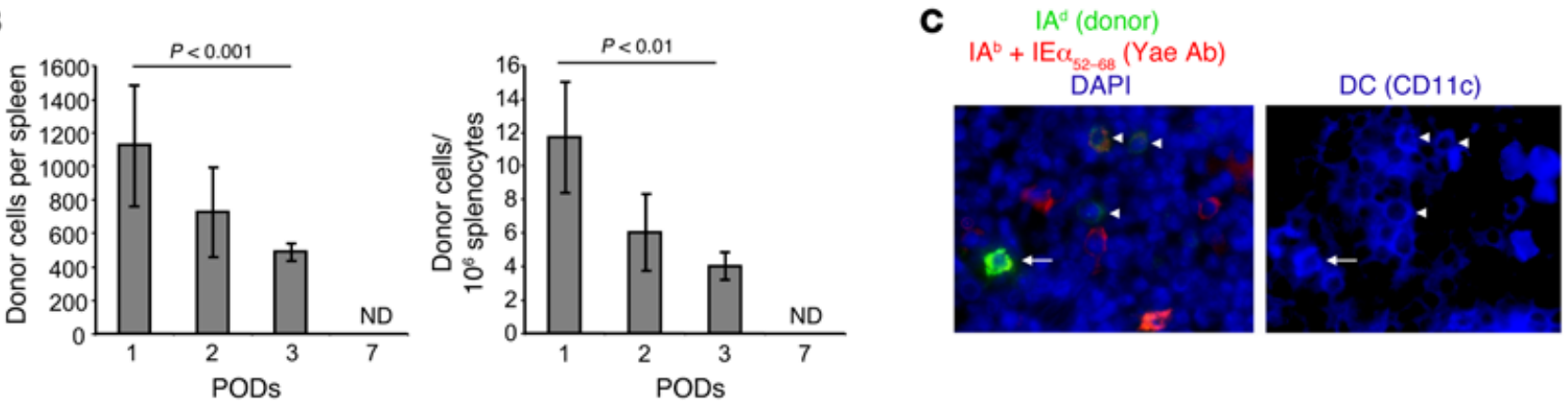

D
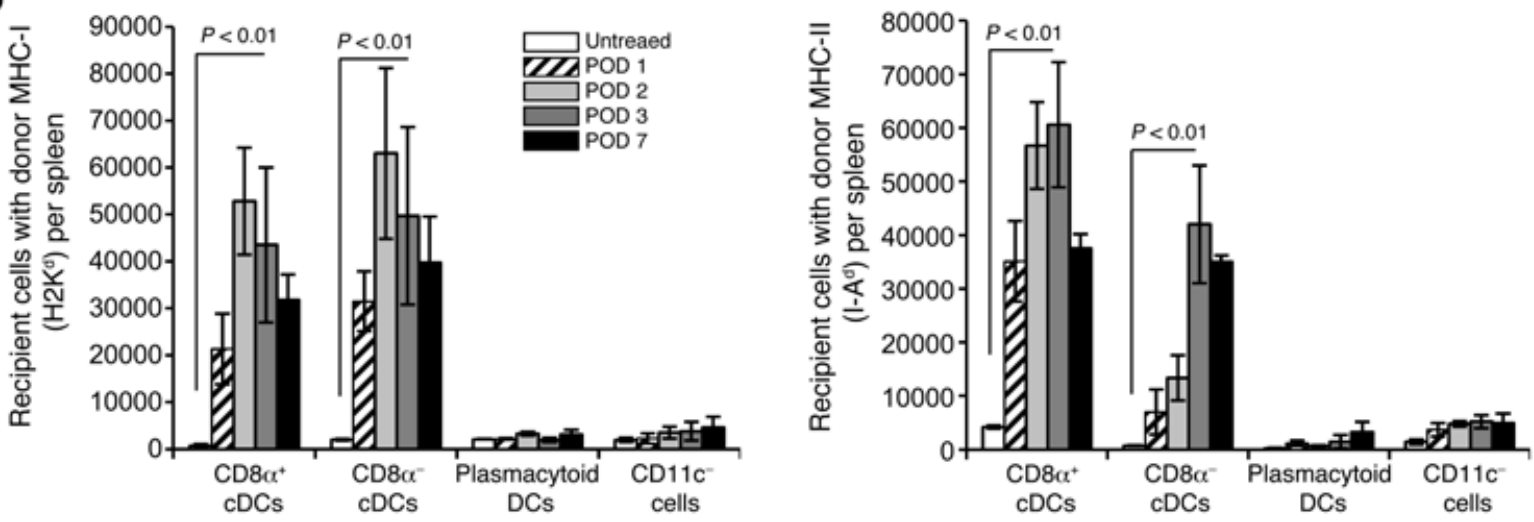

Figure 1. Transfer of donor MHC antigen in graft-draining lymphoid organs. (A) Migrating donor (BALB/C) DCs (IA $\left.{ }^{\mathrm{d}+}\right)$ in T cell areas of the recipient (B6, $\mathrm{H} 2^{b}$ ) spleen (arrows, insets). Confocal microscopy, original magnification, $\times 200$. BALB/c CDCs (CD45.2 $2^{+}$CD45.1-CD11 $\mathrm{c}^{+}$) homed in the recipient (B6) spleen were undetectable by flow cytometry. Numbers indicate percentages of cells in the corresponding quadrant. Dot plots are representative of 1 untreated or recipient mouse of 3 per time point. (B) Quantities of donor cells mobilized from BALB/c heart grafts to the recipient (B6) spleens estimated by genomic PCR. Mean \pm SD, 3 mice per variable. ND, not detected. (C) A donor DC (IA dhi arrow) in the recipient spleen next to DCs expressing IA dim or the IA (B6)-IE $\alpha_{52-68}(B A L B / c)$ complex (arrowheads, detected with the Yae $A b$ ) that likely corresponded to recipient DCs that acquired donor IAd or donor IE $\alpha_{52-68}$ peptide, respectively. Confocal microscopy, original magnification, $\times 200$. (D) FACS analysis of numbers of recipient (B6) splenic APCs with donor H2K ${ }^{d}$ or IA ${ }^{d}$ molecules at successive PODs after transplantation of BALB/c hearts. Mean \pm SD, 3 mice per variable. $P$ values were generated by 1-way ANOVA followed by Tukey-Kramer multiple comparisons test.

to a higher number of recipient APCs in graft-draining lymphoid tissues. They also unveil at the ultrastructural level and in vivo the mechanism of cross-dressing of recipient cDCs with donor MHC molecules after transplantation and the primary role that this pathway has in allograft rejection.

EVs, including exosomes, are emerging as potential biomarkers and therapeutic agents in transplantation medicine (17). Although increasing evidence suggests that EVs may have relevant biological functions, the information in vivo is still very limited (18). Our results reveal in vivo a rather unexpected role for donor-derived exosomes in the pathophysiology of transplant rejection, and provide an explanation for the potency of alloimmunity. A deeper understanding of how such mechanisms operate in situ will open new venues for development of therapies to treat transplant rejection.

\section{Results}

Donor leukocytes from transplanted hearts home in low numbers in lymphoid tissues. It is assumed that donor DCs migrated from allografts sensitize directly alloreactive T cells in lymphoid organs $(1,2)$. However, we found that after heterotopic (abdomen) transplantation of fully mismatched BALB/c ( $\left.2_{2}{ }^{\mathrm{d}}, \mathrm{CD} 45.2\right)$ hearts in $\mathrm{C} 57 \mathrm{BL} / 6\left(\mathrm{~B} 6 ; \mathrm{H} 2^{\mathrm{b}}, \mathrm{CD} 45.1\right)$ mice, donor DCs were difficult to detect by microscopy and undetectable by flow cytometry in the spleen (Figure 1A). When quantified by PCR, a maximum of 1,127 
A

Gate: CD11 ${ }^{\text {high }}$ splenic recipient's cDCs (CD45.1+ CD45.2-)

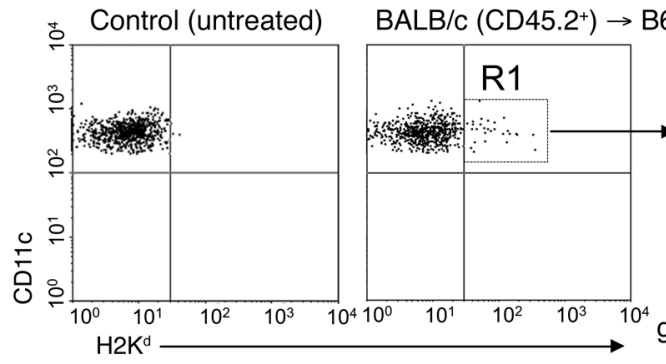

6 CD45.1+ (POD3)

FACS-sorting

Labeling with $5 \mathrm{~nm}$ gold-H2D ${ }^{d}-1 A^{d} A b$ alone or plus $12 \mathrm{~nm}$ gold-CD9 or CD63 Ab

Analysis by TEM
B

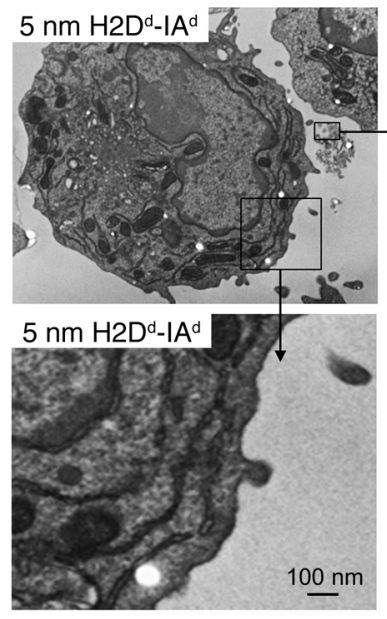

$5 \mathrm{~nm} \mathrm{H} 2 \mathrm{D}^{\mathrm{d}}-1 \mathrm{~A}^{\mathrm{d}}$
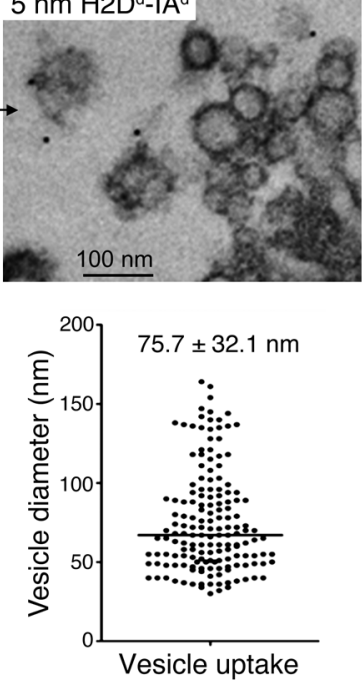

C
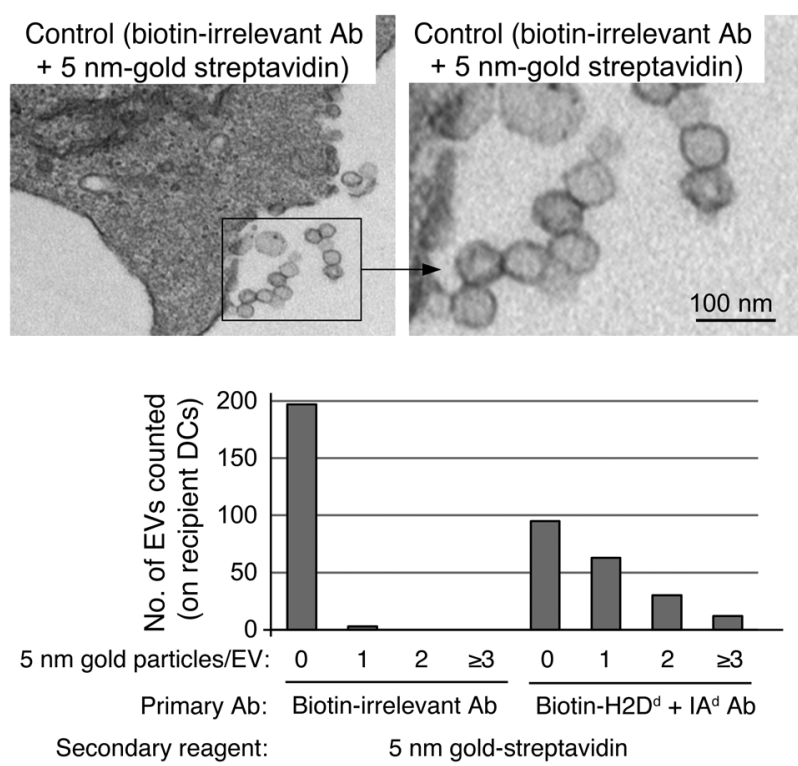

D
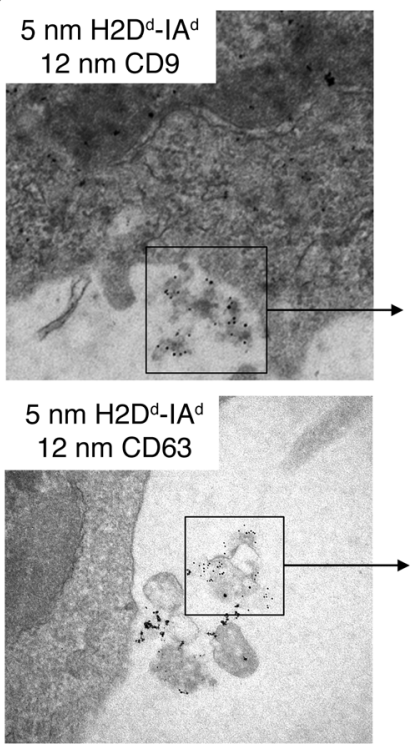

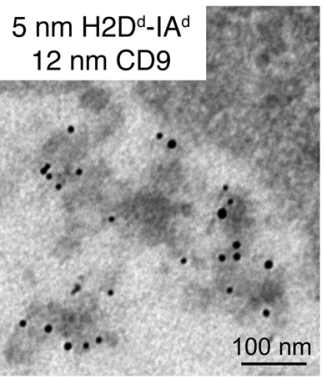

$5 \mathrm{~nm} \mathrm{H} 2 \mathrm{D}^{\mathrm{d}}-\mathrm{I} \mathrm{A}^{\mathrm{d}}$ 12 nm CD63

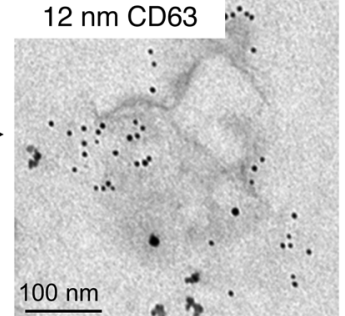

Figure 2. Recipient CDCs acquire donor MHC molecules through EVs. (A) FACS sorting of recipient (B6) CDCs bearing donor $\mathrm{H} 2 \mathrm{~K}^{\mathrm{d}}$, analyzed 3 days after transplantation of BALB/c hearts. (B) Transmission EM (TEM) image of a recipient cDC, sorted in R1 of $\mathbf{A}$, showing EVs carrying donor H2D and IA ${ }^{d}$. The area in the small rectangle is shown at higher magnification on the right. Recipient CDCs did not express donor (BALB/c) MHC directly on the surface - a section of the plasma membrane is shown at higher magnification below, original magnification, $\times 2,500-\times 10,000$. One image representative of 60 cells analyzed with gold-labeled EVs attached. Dot plot: size of EVs attached to recipient cDCs. (C) Recipient CDC, FACS-sorted in R1 of $\mathbf{A}$, and labeled with

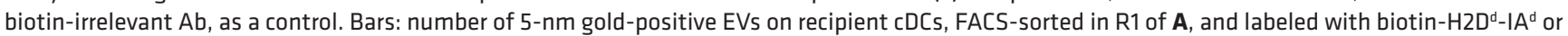
control biotin-irrelevant Abs. (D) Cluster of EVs, attached to a recipient (B6) CDC, FACS-sorted in R1 of A, expressing donor H2D and IAd, and CD9 or CD63. One image representative of 50 cells analyzed with gold-labeled EVs attached.

\pm 372 donor cells was detected in the spleen on postoperative day (POD) 1 (Figure 1B). In the retroperitoneal and mediastinal lymph nodes of the recipients, donor DCs were also difficult to detect by microscopy, and were undetectable by quantitative genomic PCR (detection limit of $1 \mathrm{BALB} / \mathrm{c}$ cell in $10^{6} \mathrm{~B} 6$ cells), during the first 7 PODs (Supplemental Figure 1, A-C; supplemental material available online with this article; doi:10.1172/JCI84577DS1). Therefore, we postulated that the donor leukocytes that reach the graftdraining lymphoid tissues must transfer via cross-dressing donor MHC to a high number of recipient APCs. Indeed, we detected donor MHC on recipient splenic cDCs by microscopy and FACS analysis (Figure 1, C and D). Surprisingly, FACS analysis revealed that the quantity of recipient splenic APCs carrying donor MHC was approximately 100-fold higher than the number of donor DCs homed to the spleen (Figure 1, B and D, and Supplemental Figure 2). Donor MHC molecules were found mainly on recipient cDCs (Figure 1D). Thus, after transplantation of fully mismatched cardiac grafts, donor DCs were detected in limited numbers in draining lymphoid tissues, whereas a higher number of recipient cDCs carried donor MHC molecules on the cell surface.

Graft DCs transfer EVs with functional MHC antigens to recipient APCs in lymphoid tissues. Next, we investigated the mechanism(s) 
A
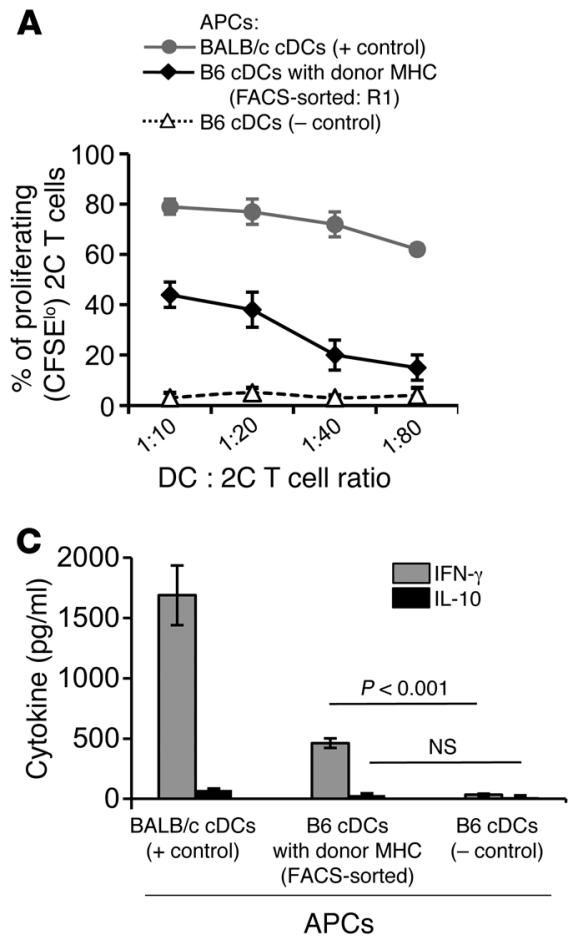

B
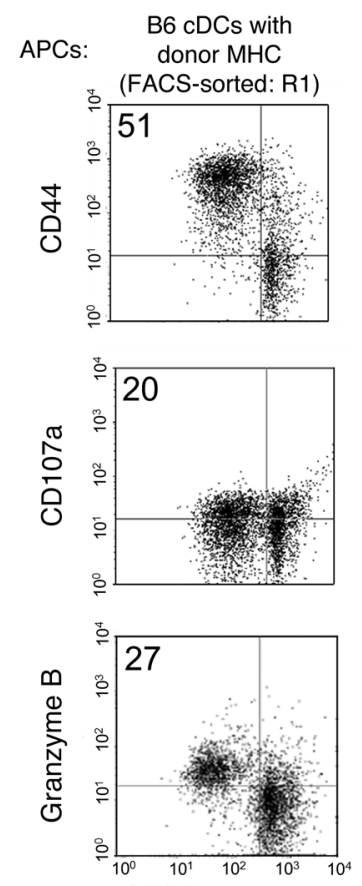

CFSE
Cell gate: $\mathrm{CD}^{+} \mathrm{CD}^{+}$cells $(2 \mathrm{C}$ T cells)
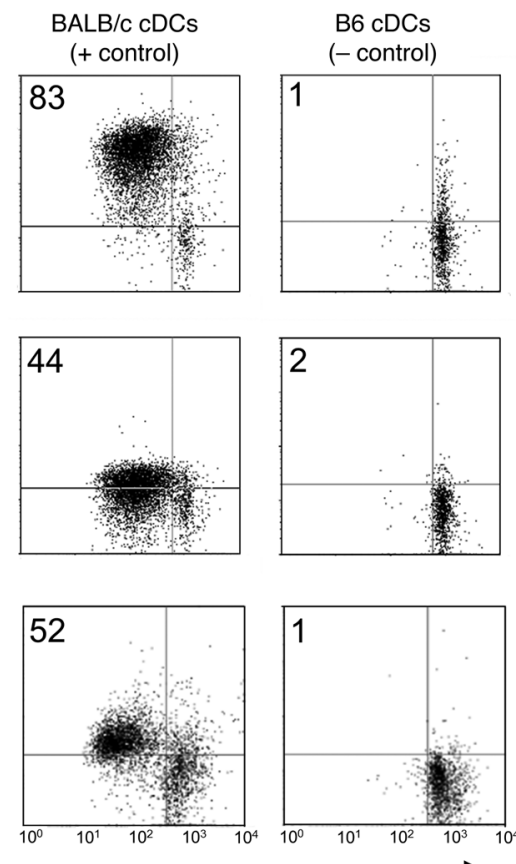

Figure 3. Recipient CDCs that acquire intact donor MHC molecules in vivo promote T cell immunity. (A) Recipient CDCs carrying donor MHC (FACS-sorted in R1 of Figure 2A) triggered proliferation of 2C CD8 T cells against BALB/C H2L ${ }^{d}$. (B and C) Expression of T cell activation/effector markers (B) and cytokine secretion (C) of 2C T cells following stimulation by recipient (B6) splenic CDCs carrying donor MHC (FACS-sorted in R1 of Figure 2A), or by control cDCs.

Numbers in dot plots indicate percentages of cells in the corresponding quadrants. IL-5 and IL-17A were undetectable by ELISA. In A-C, 1 representative of 4 experiments is shown. $P$ values were generated by 1-way ANOVA followed by Tukey-Kramer multiple comparisons test.

by which recipient $\mathrm{CDCs}$ acquire donor $\mathrm{MHC}$ molecules in graftdraining lymphoid tissues using B6 (CD45.1) mice with transplanted BALB/c (CD45.2) hearts. On POD 3, recipient splenic cDCs $\left(\mathrm{CD} 45.1^{\text {hi }} \mathrm{CD} 45.2^{-} \mathrm{CD} 11 \mathrm{c}^{\text {hi }}\right.$ cells) (Supplemental Figure 2) bearing donor $\mathrm{H}_{2} \mathrm{~K}^{\mathrm{d}}$ were FACS-sorted, and examined by electron microscopy (EM) (Figure 2A). We found that donor MHC was transferred via EVs attached to recipient $\mathrm{cDCs}$ (Figure 2B). The EVs were $75.7 \pm 32.1 \mathrm{~nm}$ in diameter and positive for the exosomeassociated tetraspanins CD9 and CD63 (Figure 2, B-D). Exosomes are EVs of endocytic origin released by fusion of multivesicular bodies (MVBs) with the cell membrane (13-16). We did not find donor-derived EVs fusing with recipient $\mathrm{CDCs}$, or donor MHC molecules expressed directly on the recipient $\mathrm{CDC}$ surface (Figure 2B). The donor-derived EVs did not incorporate into the plasma membrane of the acceptor (B6) cDCs, but remained attached to it, partially preserving its vesicular structure. Importantly, the lack of expression as an integral membrane protein of donor $\mathrm{H}_{2} \mathrm{D}^{\mathrm{d}}$ and IA ${ }^{\mathrm{d}}$ molecules on the surface of those cDCs cross-dressed with the EVs confirmed the recipient (B6) origin of the acceptor cDCs (Figure 2B). FACS-sorted recipient (B6) splenic cDCs carrying donor $(\mathrm{BALB} / \mathrm{c}) \mathrm{MHC}$ activated naive $2 \mathrm{C} \mathrm{CD} 8 \mathrm{~T}$ cells, which are directly alloreactive to BALB/c H2L (Figure 3, A-C). Thus, recipient cDCs cross-dressed in vivo with EVs carrying donor MHC molecules can prime directly alloreactive $\mathrm{CD} 8 \mathrm{~T}$ cells.

To gain further insight into the MHC antigen cross-dressing process, we investigated the mechanism of MHC transfer between cDCs through EVs in cocultures of BALB/c cDCs (CFSE-labeled) and acceptor B6 cDCs (Figure 4A). Preparations of FACS-sorted cDCs demonstrated that acquisition of BALB/c MHC by B 6 cDCs in vitro occurred through $\mathrm{CD} 9^{+} \mathrm{CD} 63^{+} \mathrm{EVs} 74.7 \pm 38.2 \mathrm{~nm}$ in diameter (Figure 4, A and B). Release of similar donor (BALB/c) EVs into the gap between BALB/c and B6 cDCs was detected in pellets of $\mathrm{CDC}$ cocultures (Supplemental Figure 3). The average size of the EVs transferred from the BALB/c DCs was similar to that of intraluminal vesicles located in MVBs of BALB/c DCs (Supplemental Figure 4). The transferred EVs remained attached to or were internalized by the target cDCs, but did not fuse with the cell membrane of acceptor cDCs (Figure 4, A and C). Mature BALB/c cDCs transferred more $\mathrm{H}_{2} \mathrm{~K}^{\mathrm{d}}$ and $\mathrm{IA}^{\mathrm{d}}$ than freshly isolated cDCs, and donor MHC passage was detectable up to a ratio of 1 donor to 32 acceptor cDCs (Figure 5A). BALB/c cDCs failed to transfer MHC when separated from B6 cDCs by $0.4-\mu \mathrm{m}$-pore transwells (Figure 5B), which confirms that MHC transfer occurred via EV clusters that, because of their hydrodynamic size (i.e., Stokes radius), did not cross the filter pores. Thus, in the context of a passive Brownian-like diffusion, EV clustering might play a key role in the lack of migration of the vesicles through a $0.4-\mu \mathrm{m}$ pore.

B6 cDCs that acquired BALB/c EVs in cDC cocultures activated directly alloreactive $2 \mathrm{C} \mathrm{T}$ cells (Supplemental Figure 5). Controls demonstrated that $2 \mathrm{C}$ T cell stimulation was not due to carryover of BALB/c cDCs to the FACS-sorted B6 cDCs crossdressed with BALB/c EVs (Supplemental Figure 5). The fact that the same $\mathrm{B} 6 \mathrm{cDCs}$ also primed $1 \mathrm{H} 3.1 \mathrm{CD} 4 \mathrm{~T}$ cells specific for the BALB/c-derived IE $\alpha_{52-68}$ peptide presented by (B6) IA ${ }^{\mathrm{b}}$ (Supple- 
A
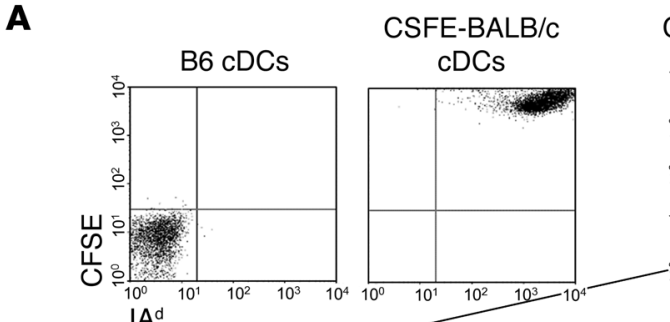

$\mathrm{CFSE}^{+} \mathrm{BALB} / \mathrm{C} \mathrm{cDCs}$
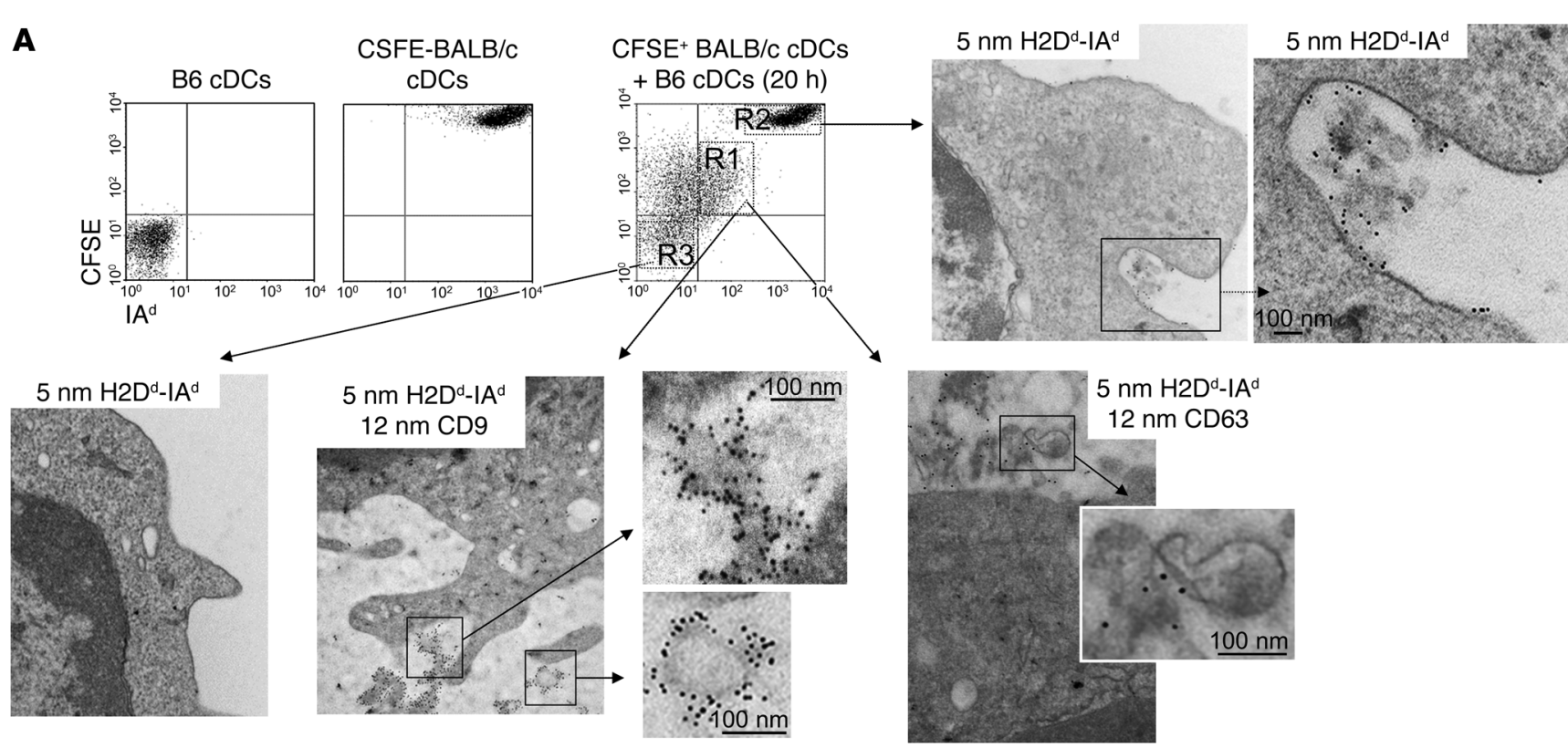

B
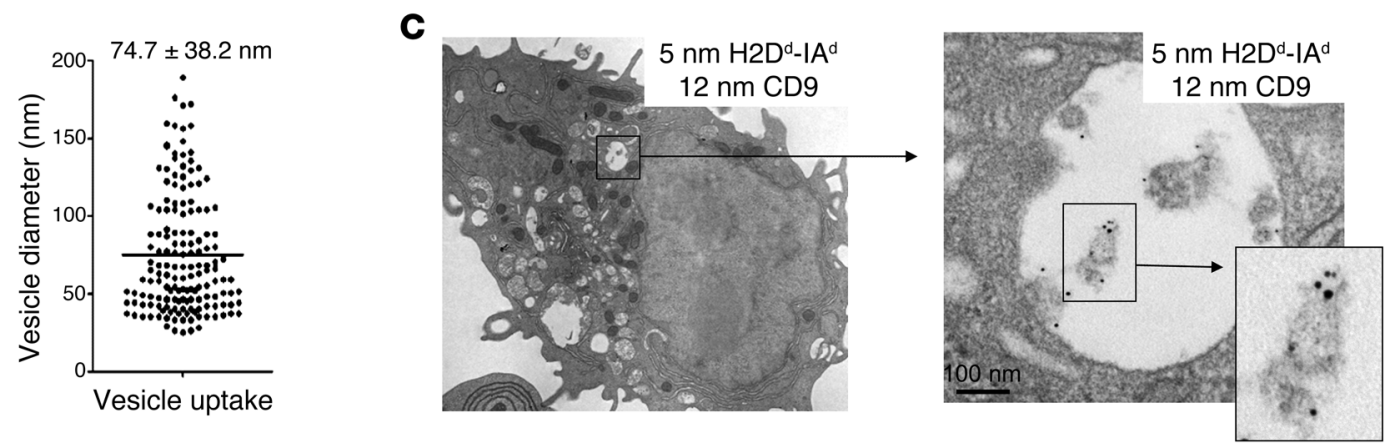

Figure 4. Passage of MHC molecules via transfer of exosomes between DCs in vitro. (A) Passage of $I A^{d}$ from CFSE-labeled $B A L B / C$ cDCs to $B 6$ cDCs in vitro (analyzed by FACS). Images of CDCs, FACS-sorted in R1, showing (BALB/C) H2D and IA transferred to (B6) CDCs through EVs expressing CD9 and CD63. $\mathrm{R} 2$ : $B A L B / c$ (donor) $c D C s$ releasing exosomes bearing $\mathrm{H}_{2} \mathrm{D}^{\mathrm{d}}$ and IAd (rectangle). R3: Recipient $c D C s$ do not express donor MHC on their surface. Results are representative of 3 experiments. Transmission EM, original magnification, $\times 20,000-\times 60,000$. Images are representative cells from 4 independent experiments. (B) Size of EVs bearing BALB/c H2D and IA ${ }^{d}$, and attached to B6 CDCs. (C) Images of EVs expressing BALB/c H2D and IAd, and CD9, which have been internalized by (B6) cDCs. Recipient CDCs were FACS-sorted in R1 (A), then labeled with biotin-H2Kd and -IAd Abs plus CD9 Ab, followed by goldconjugated secondary reagents, and then maintained at $37^{\circ} \mathrm{C}$ (30 minutes) to promote internalization of the EVs. Original magnification, $\times 20,000-$ $\times 80,000$. A representative single cell from 1 of 2 independent experiments is shown.

mental Figure 6) supports our finding by EM that donor EVs are also internalized by recipient APCs for alloantigen processing for indirect presentation to $\mathrm{T}$ cells (Figure $4 \mathrm{C}$ ).

To identify the type(s) of EVs that transfer MHC, cocultures of BALB/c and B6 DCs were done using donor (BALB/C) DCs pretreated with Rab27a siRNA to inhibit exosome release (19), or in the presence of imipramine or DEVD, which decrease release of microvesicles (MVs) or apoptotic EVs, respectively (20-22) (Supplemental Figures 7 and 8). Transfer of IAd from BALB/c to B6 cDCs was reduced only by treatment of donor DCs with Rab27a siRNA (Figure 5C), which indicates that in our model, exosomes play a key role in the transfer of donor MHC antigens to recipient APCs.

Graft DCs transfer EVs to recipient APCs upon arrival in graftdraining lymphoid tissues. Our findings suggest that donor leukocytes from the graft must first home to the lymphoid tissue, and then transfer EVs carrying donor MHC to recipient APCs. To test this idea, BALB/c bone marrow-derived DCs (BMDCs) express- ing red fluorescent protein (RFP) linked to the exosome-associated tetraspanin CD63 (CD63-RFP BMDCs) (Supplemental Figure 9), and with the nuclei labeled blue (Hoechst), were injected in the footpad or tail vein of CD11c-yellow fluorescent protein (CD11c-YFP) B6 mice. After 16 hours, the popliteal lymph nodes and spleen were analyzed by confocal microscopy. Although individual exosomes range in size between 30 and $100 \mathrm{~nm}$ and therefore are below the resolution limit of conventional confocal microscopy, we have demonstrated that exosomes are transferred between DCs in clusters (Figure 2B) that are detectable by our imaging system. In lymph nodes (Figure 6, A-C, and Supplemental Videos 1-3) and spleen (Figure 6, D and E, and Supplemental Videos 4 and 5), CD63-RFP BMDCs transferred $\mathrm{RFP}^{+} \mathrm{EVs}$ to lymphoid tissue $\mathrm{YFP}^{+} \mathrm{cDC}$. The $\mathrm{RFP}^{+} \mathrm{EV}$ s were retained on the surface of $\mathrm{YFP}^{+} \mathrm{cDC}$ up to a follow-up period of 4 hours (Supplemental Video 6), or were internalized (Figure 6, B-E, and Supplemental Videos 3, 5, and 7). By contrast, we detected minimal 
A

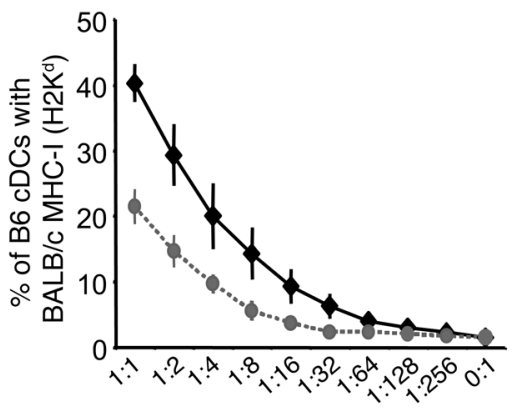

Donor DC : acceptor DC ratio

B

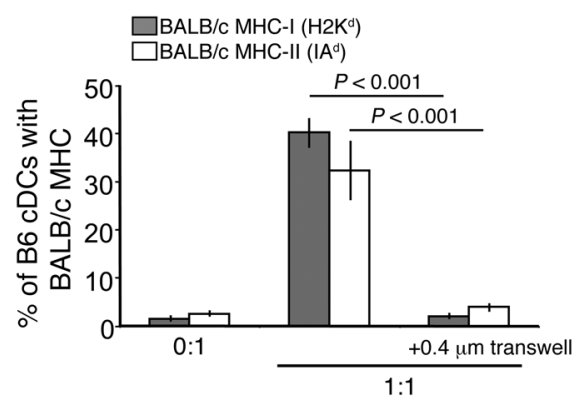

Donor DC: acceptor DC ratio

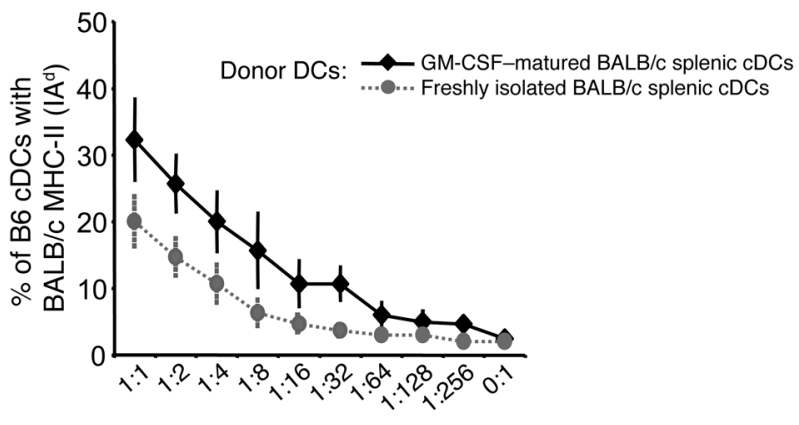

Donor DC : acceptor DC ratio

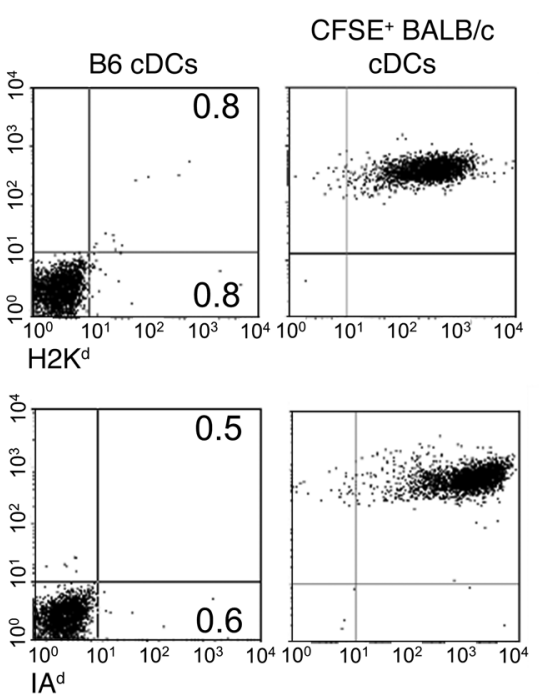

CFSE ${ }^{+}$BALB/c cDCs + B6 cDCs
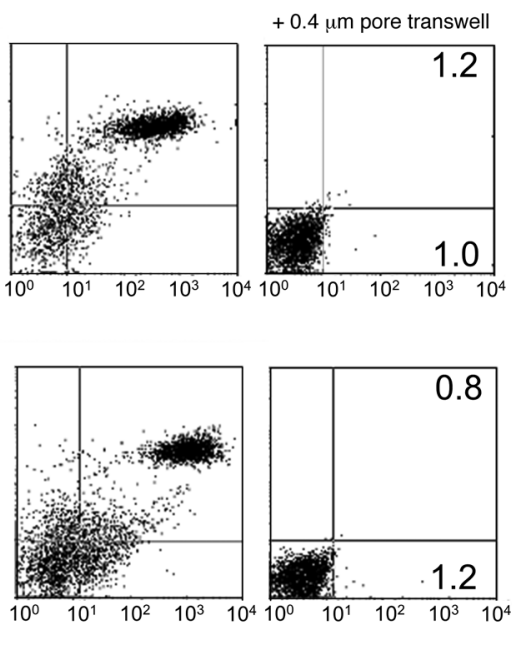

C

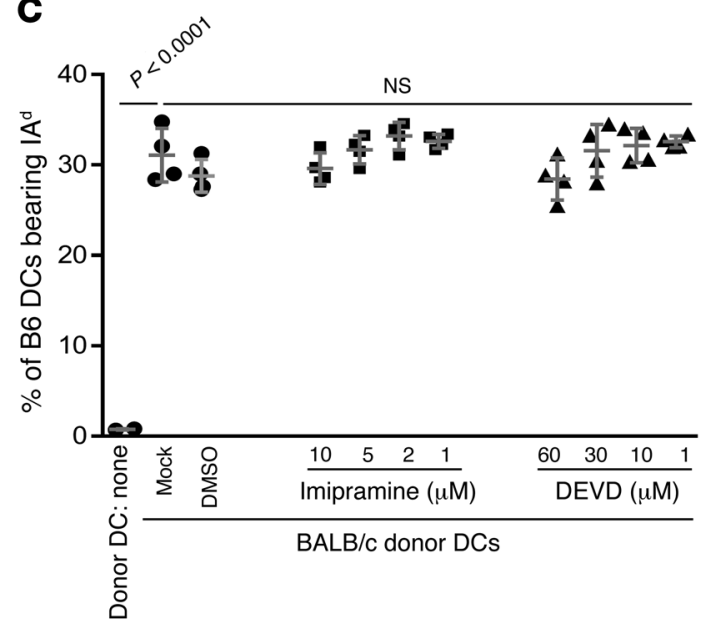

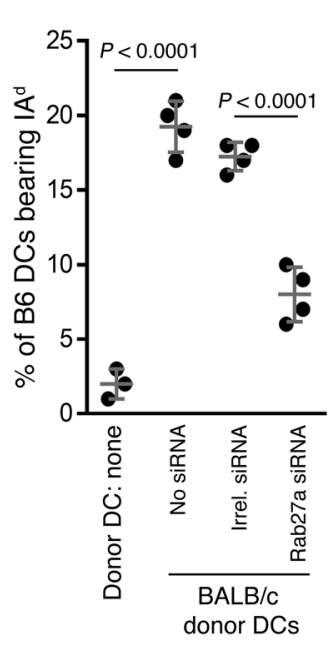

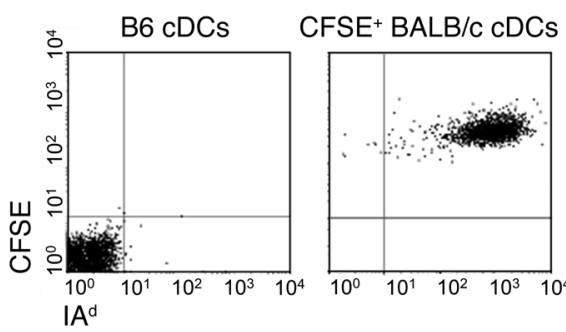

$\mathrm{IA}^{\mathrm{d}}$

$\mathrm{CFSE}^{+}$irrel. siRNA $\mathrm{CFSE}^{+}$Rab27a

BALB/c DCs siRNA BALB/c DCs

$+\mathrm{B} 6 \mathrm{cDCs}+\mathrm{B} 6 \mathrm{cDCs}$

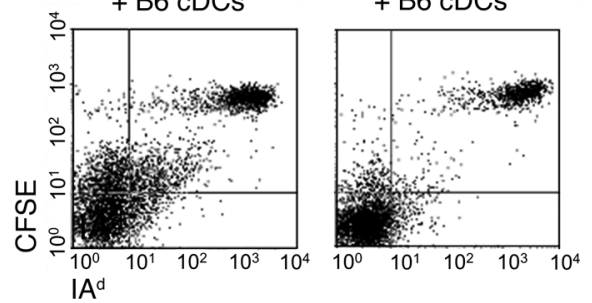

Figure 5. Efficiency of transfer of intact MHC molecules via exosomes between DCs. (A) Transfer efficiency of H2K and IAd from BALB/C to B6 cDCs (analyzed by FACS). Mean \pm SD. One representative experiment of 2. (B) Percentages of B6 cDCs that acquired H2K and IA from BALB/c cDCs following culture with BALB/C CDCs together, or separated by $0.4-\mu \mathrm{m}$-pore transwells (analyzed by FACS). Results are representative of 4 experiments. Numbers in dot plots indicate percentages of cells in the corresponding quadrant. (C) Effect of imipramine or DEVD added to the culture medium, or pretreatment of donor $B A L B / c$ DCs with Rab27a siRNA, on transfer of IA ${ }^{d}$ from BALB/c DCs to B6 cDCs in vitro (analyzed by FACS). Results from 1 representative of 4 independent experiments are shown. $P$ values were generated by 1-way ANOVA followed by Tukey-Kramer multiple comparisons test. 

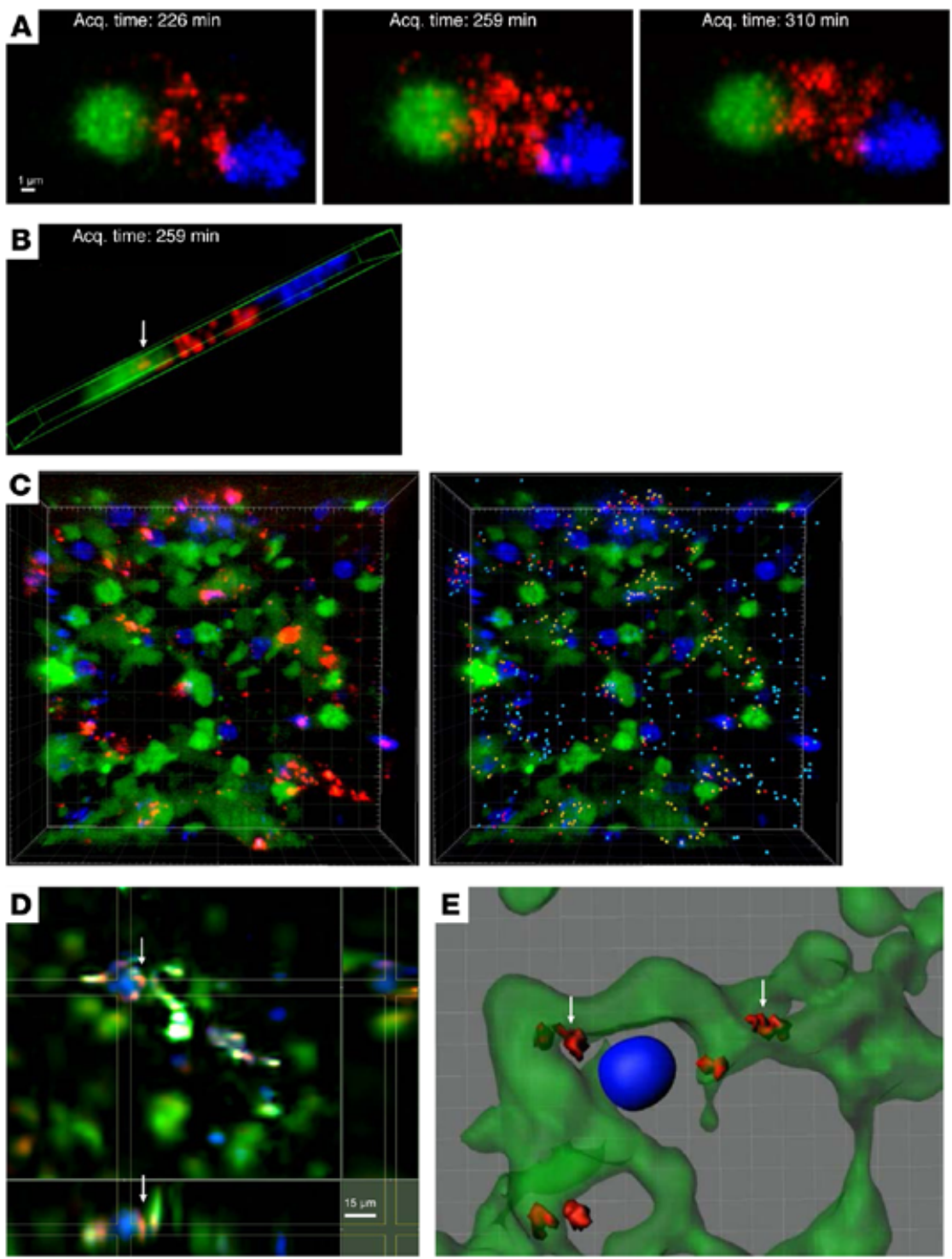

Figure 6. Recipient cDCs acquire clusters of donorderived exosomes in vivo. (A) Time-lapse analysis (confocal) of transfer of RFP-tagged exosome clusters from a migrating CD63-RFP BALB/C DC (blue nucleus + RFP-exosomes) to a lymph node cDC (YFP', in green). Scale bar: $1 \mu \mathrm{m}$. (B) Side view of $\mathbf{A}$. The arrow indicates internalized RFP-tagged EVs. (C) Left: 3D panoramic view (confocal) of a lymph node of a CD11c-YFP B6 mouse injected with CD63-RFP BALB/c DCs. Right: RFP-exosome clusters in relationship to host $\mathrm{YFP}^{+} \mathrm{CDCs}$, on the same image analyzed with Imaris X64. Red dots: RFP-exosomes on YFP+ B6 CDCs. Yellow dots: RFPexosomes internalized by $\mathrm{FFP}^{+} \mathrm{B} 6 \mathrm{CDCs}$. Blue dots: RFP-exosomes in CD63-RFP BALB/C DCs, free or

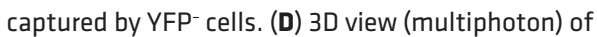
the spleen of a CD11c-YFP B6 mouse injected i.v. with CD63-RFP BALB/c DCs. Arrow: interaction between injected CD63-RFP BALB/C DCs and host YFP ${ }^{+}$ cDCs. Scale bar: $15 \mu \mathrm{m}$. (E) Analysis (Imaris X64) of transfer (arrows) of RFP-exosome clusters from a CD63-RFP BALB/C DC to host YFP+ ${ }^{+}$CDCs (in green) in the spleen. In $\mathbf{A}-\mathbf{E}$, results are representative of 4 independent experiments. transfer of RFP to $\mathrm{YFP}^{+} \mathrm{cDCs}$ after injection of control BALB/c BMDCs expressing cytosolic RFP (Supplemental Video 8), which should be able to transfer MVs or apoptotic blebs containing cytosolic RFP within the EVs. These results indicate that migrating DCs transfer clusters of exosomes to cDCs in lymphoid organs in vivo, and that such EVs remain attached to the cell surface or are internalized by the acceptor DCs.

Acquisition of EVs released by graft migrating DCs promotes recipient APC activation. We found that recipient splenic $\mathrm{CDCs}$ upregulate activation markers after transplantation of $\mathrm{BALB} / \mathrm{c}$ hearts in B6 mice (Figure 7A). Therefore, we investigated whether passage of EVs promotes DC activation. Following injection of CD63-RFP $\mathrm{BALB} / \mathrm{c}$ BMDCs in CD11c-YFP B6 mice, those splenic cDCs that acquired RFP-tagged exosomes expressed higher amounts of endogenous MHC class II (IA ${ }^{\text {b }}$, CD40, CD80, and CD86, but similar levels of the regulatory molecule PD-L1, in comparison with splenic cDCs without RFP (Figure 7B). We investigated whether this phenomenon was due to preferential uptake of the exosomes by already mature cDCs, or to exosome-induced maturation of immature or semimature cDCs. The effect(s) of exosomes on DC maturation was analyzed in vitro by incubation of immature B6 BMDCs with exosomes or cell surface-shed MVs - the latter as a control $\mathrm{EV}$, released by BALB/c immature or mature BMDCs (Figure 8, A and B). We selected surface expression of endogenous MHC class II (IA $\left.{ }^{\mathrm{b}}\right)$ as a marker of B6 BMDC maturation, because other commonly used markers of DC activation such as CD86 can be transferred to the acceptor DCs through the BALB/c exosomes (Figure 8, B and C). Incubation with mature BALB/c exosomes augmented, in a dose-dependent manner, surface expression of $\mathrm{IA}^{\mathrm{b}}$ molecules on B6 BMDCs (Figure 8D). Addition of immature $\mathrm{BALB} / \mathrm{c}$ exosomes or MVs released by BALB/c mature BMDCs had little or no effect (Figure 8D). The increase of surface $\mathrm{IA}^{\mathrm{b}}$ content on B6 BMDCs required exposure to intact exosomes, since the effect disappeared when the EVs were disrupted by repetitive freezing and thawing (Figure 8D). Mature BALB/c exosomes also augmented, in a dose-dependent manner, expression of CD40, CD80, and CD86 (but not PD-L1) (Figure 9A), and the ability to stimulate third-party naive T cells in acceptor B6 BMDCs (Figure 
A Cell gate (spleen): recipient cells (CD45.1 ${ }^{+}$CD45.2-)

cDCs (CD11 $\left.\mathrm{c}^{\text {high }}\right)$ $1 c^{\text {int }} \mathrm{CD} 11 \mathrm{~b}^{-}$ Siglec $\mathrm{H}^{+}$)
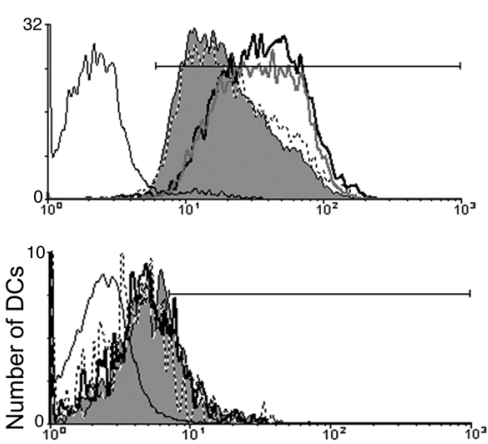

Recipient MHC class II (IA ${ }^{\text {b }}$
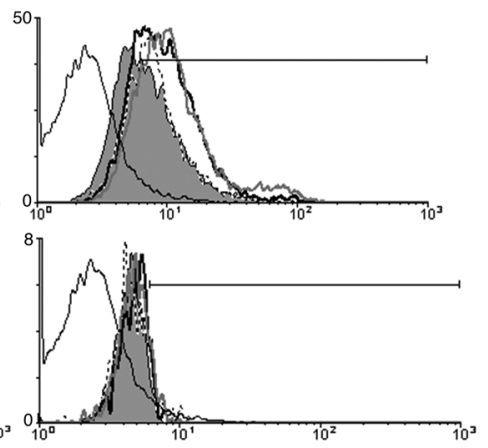

CD86
B

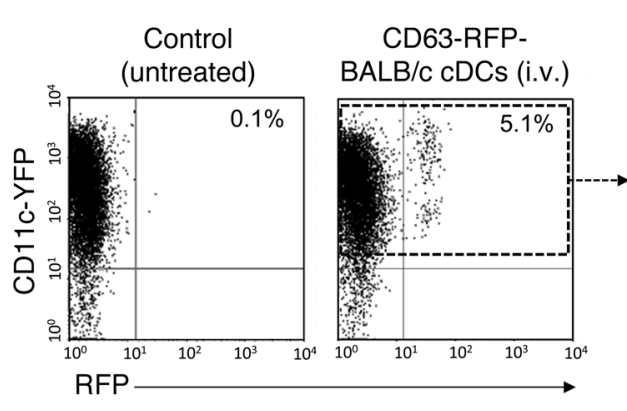

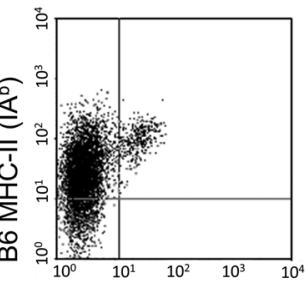
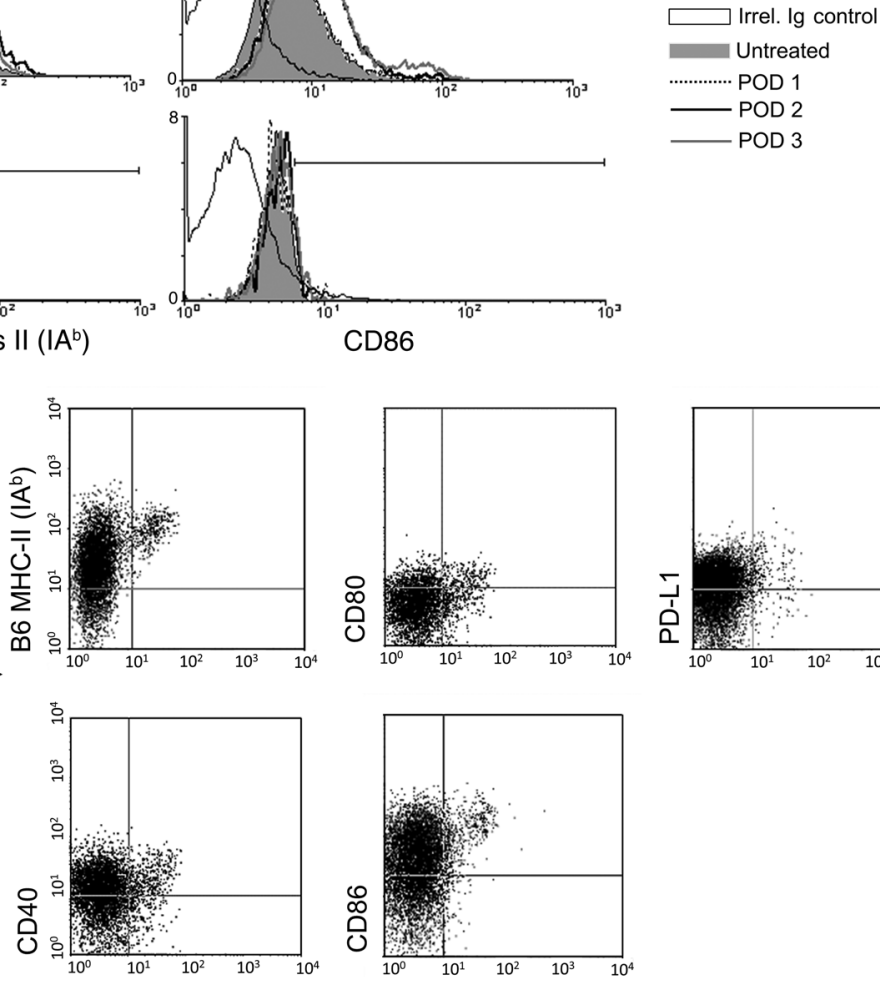

RFP
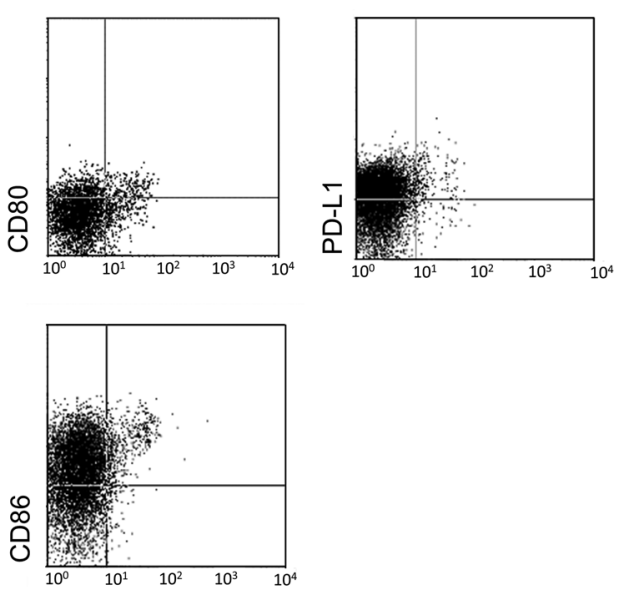

Figure 7. Transfer of exosomes promotes CDC maturation in the spleen. (A) Recipient MHC class II (B6, IA $)$ and CD86 expression by cDCs and plasmacytoid DCs (pDCs) from spleens of CD45.1 B6 mice transplanted with CD45.2 BALB/c hearts, analyzed by FACS on successive PODs. Results are representative of 3 mice per variable. (B) Effect of transfer of RFP-tagged exosomes between migrating CD63-RFP BALB/c BMDCs injected i.v. and spleen-resident cDCs of CD11C-YFP B6 mice. Numbers in dot plots indicate percentages of cells in the corresponding quadrants. CD63-RFP BALB/c BMDCs were matured by overnight incubation with IL-1 $\beta$ plus TNF- $\alpha$. Comparison by FACS analysis of expression of endogenous (B6) MHC class II (IA ${ }^{\mathrm{b}}$ ), CD40, CD80, CD86, and PD-L1 between $\mathrm{YFP}^{+} \mathrm{CDCs}$ without and with RFP+ content, analyzed 16 hours after BMDC injection. Results are from representative experiments with 4 mice per group.

9B). These results indicate that exosomes released by mature DCs promote the maturation of acceptor DCs.

Recipient cDCs present acquired donor MHC molecules to directly alloreactive $T$ cells. Since cDCs acquire donor MHC through EVs, we evaluated the role of recipient cDCs in priming directly alloreactive $\mathrm{T}$ cells. BALB/c hearts were transplanted in $\mathrm{CD} 11 \mathrm{c}-$ diphtheria toxin receptor (CD11c-DTR) B6 BM chimeras, where DT injection depletes recipient cDCs. Control CD11c-DTR-B6 chimeras not exposed to DT rejected $\mathrm{BALB} / \mathrm{c}$ heart grafts as rapidly as WT B6 recipients (Figure 10A). In contrast, survival of BALB/c cardiac grafts was significantly prolonged in CD11cDTR-B6 BM chimeras depleted of recipient cDCs by DT injection (Figure 10A). This was not due to nonspecific effects of DT, since control DT-injected WT B6 BM chimeras rejected BALB/c hearts (Figure 10A) as rapidly as untreated WT B6 recipients (not shown). The increased survival of the BALB/c grafts in DT-treated CD11c-DTR-B6 BM chimeras was not due to impaired homing of donor DCs to spleens depleted of recipient cDCs. Indeed, similar numbers of donor migrating DCs $\left(\mathrm{CD} 11 \mathrm{c}^{+} \mathrm{IA} \mathrm{A}^{\mathrm{d}}\right.$ hi) were detected by microscopy on PODs 1 and 3 in spleens of DT-treated CD11cDTR-B6 BM chimeras and control DT-injected WT B6 BM chime- ras (Figure 10B). In both cases, the few donor DCs detected were located within or next to splenic $\mathrm{T}$ cell areas (Figure 10C). Thus, the method used for depletion of recipient cDCs did not interfere with the ability of graft-derived cDCs to migrate to the spleen or to home to $\mathrm{T}$ cell areas.

$\mathrm{BALB} / \mathrm{c}$ hearts transplanted in DC-depleted CD11c-DTR-B6 chimeras exhibited less damage and inflammation than BALB/c grafts from controls (Supplemental Figure 10). As expected, the frequency of $\mathrm{T}$ cells against donor peptides presented by recipient CDCs (Figure 10D) and the titer of anti-donor antibodies (Abs) in serum (Figure 10E) (both dependent on recipient APCs) were similar to those in naive animals. Importantly, splenic $\mathrm{T}$ cells from cDC-depleted recipients did not respond against donor intact MHC molecules (Figure 10D), which indicates that recipient cDCs were necessary for sensitization of directly alloreactive $T$ cells. The results were not due to DT-induced depletion of CD11 $\mathrm{c}^{+}$ $\mathrm{T}$ cells (Supplemental Figure 11) or splenic $\mathrm{CD} 11 \mathrm{c}^{+}$macrophages (Supplemental Figure 12).

Transfer of donor MHC molecules in an orthotopic transplant model. We investigated whether transfer of donor MHC molecules through passage of exosome clusters also occurs in an orthotopic 
A
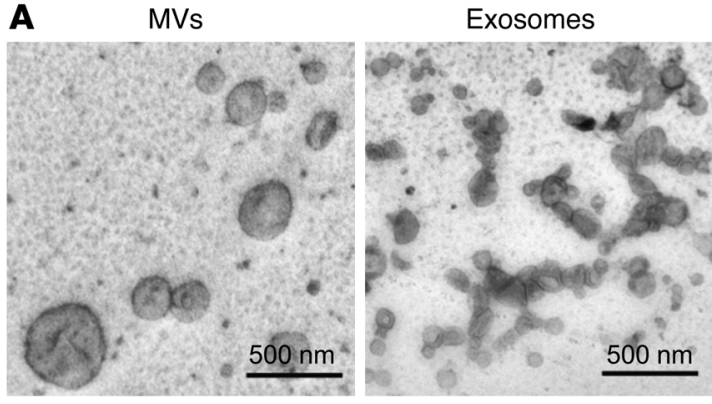

B

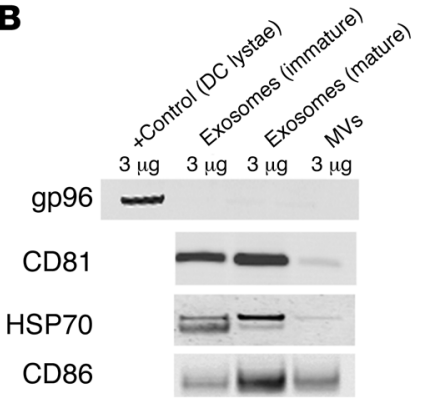

C

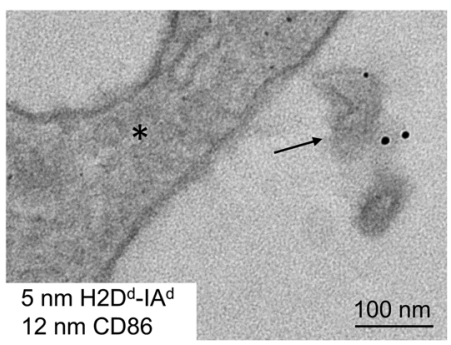

D

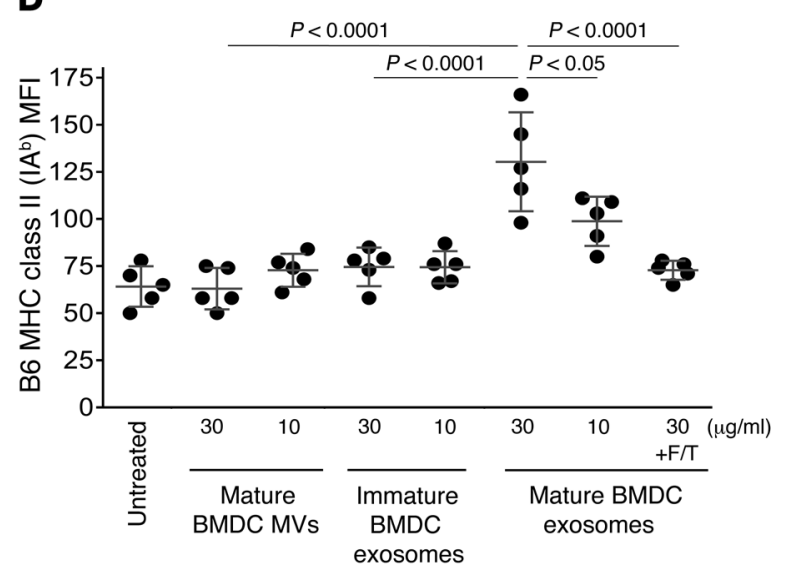

Figure 8. Effect of different EVs released by DCs on DC maturation. (A) EM analysis of MVs and exosomes secreted by BALB/C BMDCs matured by incubation with IL-1 $\beta$ and TNF- $\alpha$. Original magnification, $\times 20,000$. (B) Western blot analysis of the endoplasmic reticulum protein gp96 (control), the exosome-associated protein CD81, and the DC activation/maturation marker CD86 on different EVs released by BALB/C BMDCs. One representative Western blot of 2 is shown. (C) Detection of donor (BALB/C) MHC molecules (H2Dd-IAd) and CD86 on exosomes (arrow) from BALB/c mature BMDCs transferred to an acceptor B6 BMDC (asterisk). Transmission $E M$, original magnification, $\times 20,000$. (D) Expression of endogenous MHC class II (IA ${ }^{\text {b) }}$ (analyzed by FACS) on the surface of B6 BMDCs untreated or incubated with MVs or exosomes from BALB/c BMDCs. As a negative control, exosomes were added after 5 freeze/thaw cycles $(F / T)$. Results are from 1 representative experiment of 3 .

$P$ values were generated by 1-way ANOVA followed by Tukey-Kramer multiple comparisons test.

\section{Discussion}

After organ transplantation, donor cDCs migrate to the recipient lymphoid tissues and prime directly alloreactive $\mathrm{T}$ cells $(1,2)$. However, we found that after heterotopic (abdomen) cardiac transplantation in mice, donor DCs are detected in the spleen and graft-draining lymph nodes in low numbers, likely because, as shown before, they have short lifespan and are targets of NK cells and cytotoxic $\mathrm{T}$ cells (8-11). Besides, the transplanted heart contains a limited number of tissue-resident DCs - ranging between $11 \times 10^{4}$ and $44 \times 10^{4}$ per heart (24) - from which a reduced percentage home in recipient lymphoid tissues. Indeed, we found that, at its peak, only $1,127 \pm 372$ donor cells (mostly migrating DCs) reach the recipient spleen. This figure represents the average number of donor leukocytes at a given time, since donor leukocytes are constantly arriving and becoming apoptotic within the spleen. Beyond this potential caveat, and considering that the mouse spleen harbors approximately $20 \times 10^{6} \mathrm{~T}$ cells (Mouse Phenome Database, http:// phenome.jax.org, 2006), the ratio between donor migrating DCs and recipient $\mathrm{T}$ cells is approximately 1:20,000. Although mature DCs

transplant model of skin allografts. Following bilateral transplantation of $\mathrm{BALB} / \mathrm{c}\left(\mathrm{H} 2^{\mathrm{d}}, \mathrm{CD} 45.2\right)$ skin on the back of $\mathrm{B} 6\left(\mathrm{H} 2^{\mathrm{b}}\right.$, CD45.1) mice, we were unable to detect by FACS donor migrating cDCs in the recipient spleen (Figure 11A) and graft-draining lymph nodes (i.e., axillary and inguinal). This agrees with previous studies done in fully mismatched skin transplant models in mice $(8,23)$. During the first week after transplantation, however, a percentage of the recipient splenic $\mathrm{CDCs}$ acquired donor MHC class I $\left(\mathrm{H}_{2} \mathrm{~K}^{\mathrm{d}}+\mathrm{H} 2 \mathrm{D}^{\mathrm{d}}\right)$ and class II $\left(\mathrm{IA}^{\mathrm{d}}\right)$ molecules (Figure 11, A and B). Both $\mathrm{CD} 8 \alpha^{+}$and CD8 $\alpha^{-}$cDCs were cross-dressed with donor MHC molecules (Figure 11A). The content of donor MHC antigen per DC and the percentages of recipient $\mathrm{CDCs}$ bearing donor MHC molecules increased along with time after surgery (Figure 11B). Passage of donor MHC molecules to recipient cDCs was not detected in controls grafted with syngeneic skin (Figure 11B). By ImageStream technology (EMD Millipore), the donor $\mathrm{H} 2 \mathrm{~K}^{\mathrm{d}} / \mathrm{H} 2 \mathrm{D}^{\mathrm{d}}$ and $\mathrm{IA}^{\mathrm{d}}$ molecules were detected in spots on the recipient cDCs (Figure 11C). The punctate areas containing donor MHC molecules coexpressed the exosome marker CD63 (Figure $11 C)$, which indicates they represent clusters of donor-derived exosomes bound to the recipient cDCs. are potent APCs, their allostimulatory ability is barely detectable in mixed leukocyte cultures below a DC/T cell ratio of 1:100 (12). It is unlikely that these differences could be explained only by the 3D structure of the lymphoid tissue or mobility of naive T cells in vivo. Therefore, we investigated in this model the mechanism(s) by which the relatively few donor passenger DCs that reach the graftdraining lymphoid tissues prime so efficiently directly alloreactive $\mathrm{T}$ cells. We first noticed that although graft-derived DCs were detected at very low numbers in draining lymphoid tissues, most of the donor MHC molecules were found on clusters of EVs bound to recipient cDCs. Similar results were found in a fully mismatched orthotopic nonvascularized skin transplant model in mice.

Next, we investigated the origin of the EV-associated donor MHC molecules bound to the recipient cDCs in the draining lymphoid tissues. A possible explanation is that the graft sheds soluble MHC molecules into circulation, which are trapped by secondary lymphoid tissues. Interestingly, since its description in the 1980s $(25,26)$, soluble MHC molecules were shown to be associated with membrane fragments, which later were characterized as EVs (27). It is still uncertain whether systemic release of MHC molecules - likely through EVs - by parenchymal and 

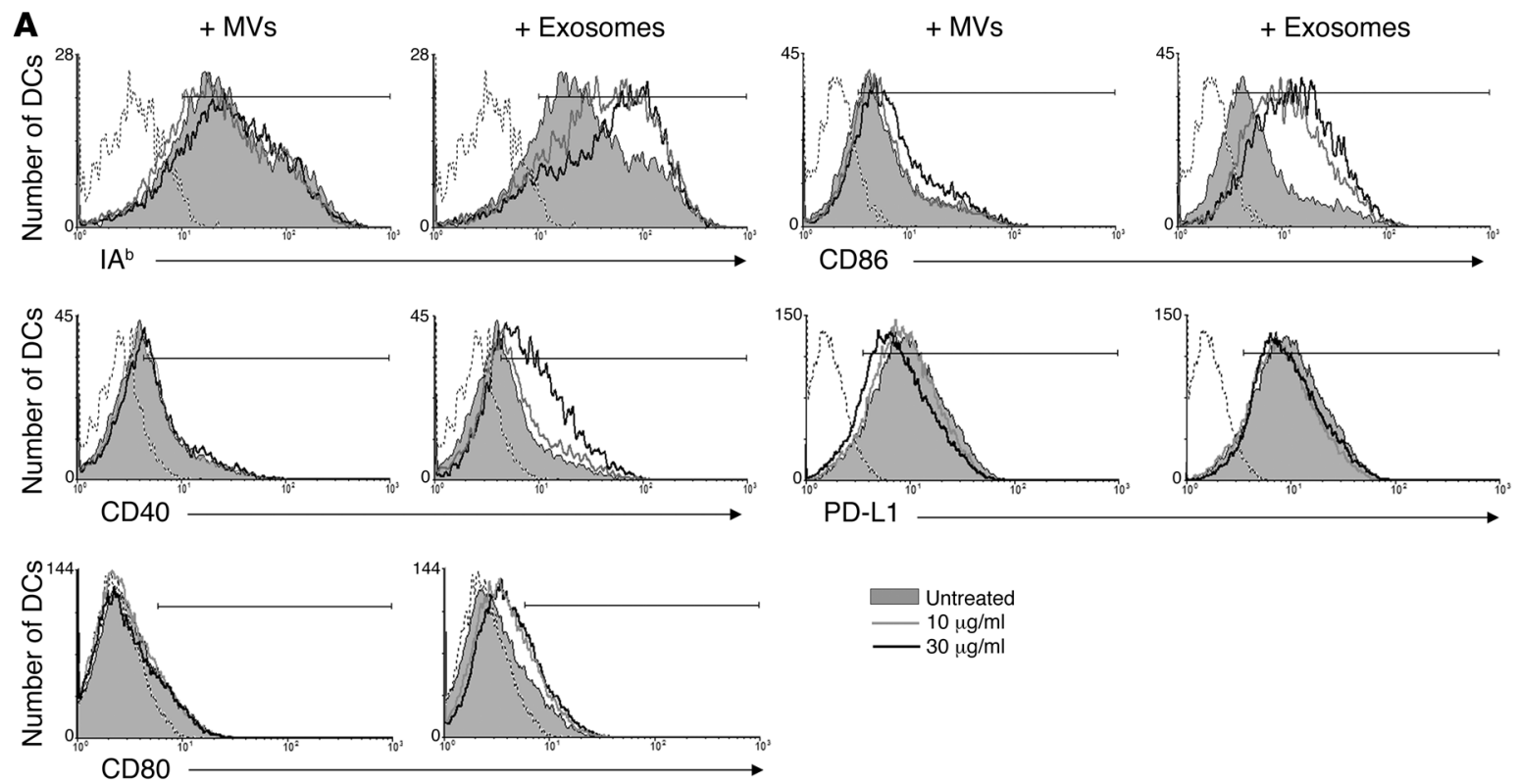

B

No APCs

B6 BMDCs
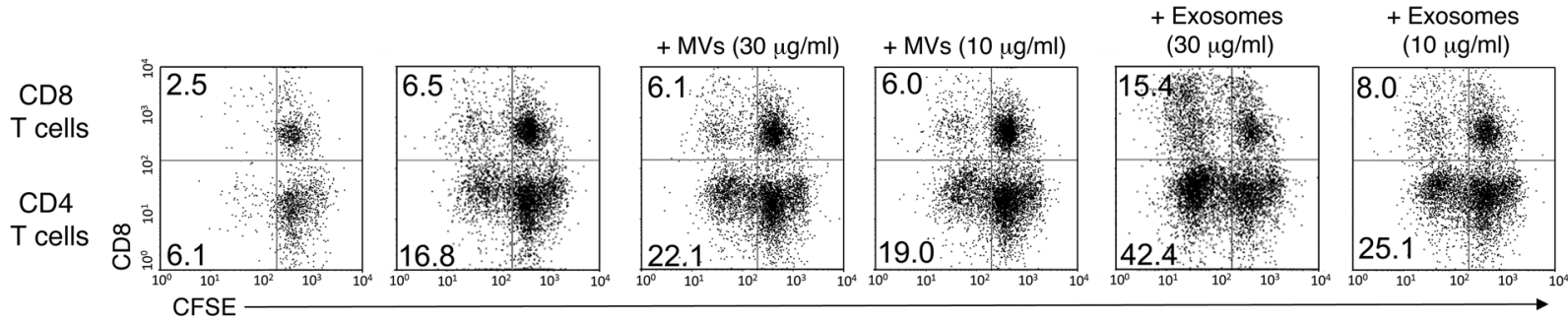

Figure 9. Transfer of exosomes released by mature DCs promotes maturation of the acceptor DCs. (A) Expression of endogenous MHC class II (IA ${ }^{b}$ ), CD40, CD80, CD86, and PD-L1 (by FACS) on B6 BMDCs untreated or incubated with MVs or exosomes from BALB/C mature BMDCs. One representative of 3 experiments is shown. (B) Ability of B6 BMDCs, untreated or exposed to MVs or exosomes from mature BALB/c BMDCs, to promote proliferation of naive (thirdparty) C3H T cells in CFSE-mixed leukocyte cultures analyzed by FACS. Numbers in dot plots indicate percentages of cells in the corresponding quadrants. In $\mathbf{A}$ and $\mathbf{B}, 1$ representative experiment of 4 is shown.

nonmigrating stromal cells of cardiac allografts is sufficient to prime directly allospecific $\mathrm{T}$ cells in graft-draining lymphoid organs. Interestingly, in the fully mismatched nonvascularized skin transplant model, we detected recipient cDCs carrying donor MHC molecules together with the exosome marker CD63 on the cell surface, but, unlike in the heart transplant model, we did not find donor DCs in the graft-draining lymphoid tissues. This finding opens the following 2 possibilities in the skin transplant model: (a) that donor exosomes are released within the graft-draining lymphoid tissue by donor migrating DCs that die rapidly after their arrival; or (b) that donor exosomes are released systemically by nonmigrating cells of the graft, or by donor DCs before leaving or trapped within the allograft. A potential problem with this latter idea is that once released systemically, the EVs passively traffic through the bloodstream and other bodily fluids, where they become dispersed, and from where they are rapidly captured for degradation by macrophages throughout the body (15). Actually, systemic dilution and rapid clearance of circulating EVs by phagocytes are drawbacks for the therapeutic application of systemically administered EVs (15).
A second possibility is that recipient monocyte-derived DCs $\left(\mathrm{CD} 11 \mathrm{c}^{\text {int }} \mathrm{CD} 11 \mathrm{~b}^{\text {hi }} \mathrm{Ly}_{6 \mathrm{C}} \mathrm{C}^{+}\right)$infiltrating the grafts take up donor MHC molecules and then migrate to lymphoid tissues to prime directly alloreactive $\mathrm{T}$ cells, or transfer donor $\mathrm{MHC}$ to recipient $\mathrm{cDCs}$. This seems unlikely, since donor MHC was detected on $\mathrm{CDCs}\left(\mathrm{CD} 11 \mathrm{c}^{\text {hi }} \mathrm{CD} 11 \mathrm{~b}^{\text {int }}\right.$ Ly6C-), instead of monocyte-derived DCs in the spleen.

A third possibility is the one described in this study, in which donor migrating DCs first reach the recipient lymphoid tissues and then transfer EVs carrying donor MHC and APC-activating signals to a higher number of recipient cDCs (28).

Previous studies have shown that leukocytes transfer MHC via mechanisms that have been termed cross-dressing $(29,30)$, trogocytosis (31), or cell nibbling (32), depending on the model and type of cell involved. In the transplantation field, Lechler and colleagues originally described cross-dressing of DCs with allogeneic MHC molecules as the basis of what they termed the semidirect pathway of allorecognition (33). Since then, others have detected in mouse models the presence of donor MHC molecules on recipient APCs after heart and kidney transplantation, and even the reversed passage of host MHC molecules to donor 

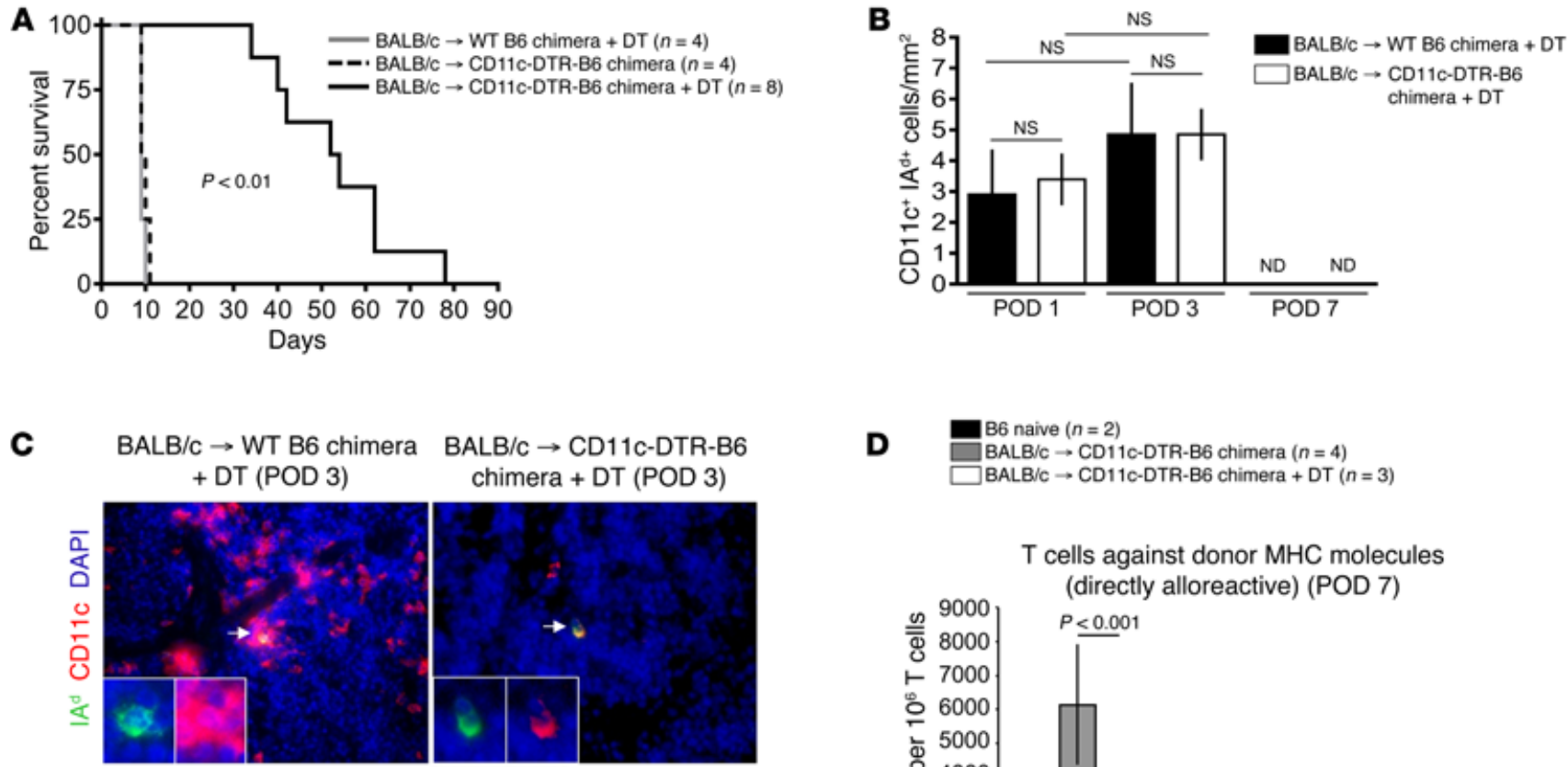

\section{$\mathrm{BALB} / \mathrm{c} \rightarrow \mathrm{CD} 11 \mathrm{c}-\mathrm{DTR}-\mathrm{B} 6$ chimera + DT (POD 3)}
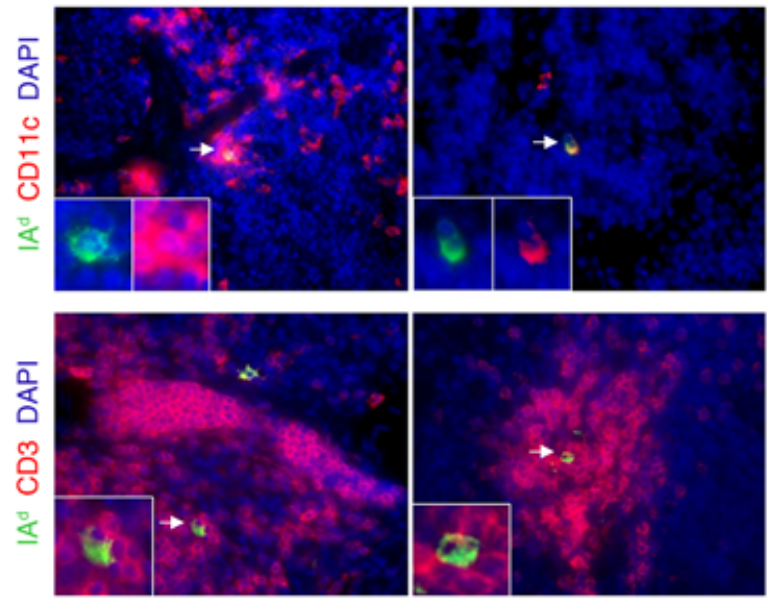

E
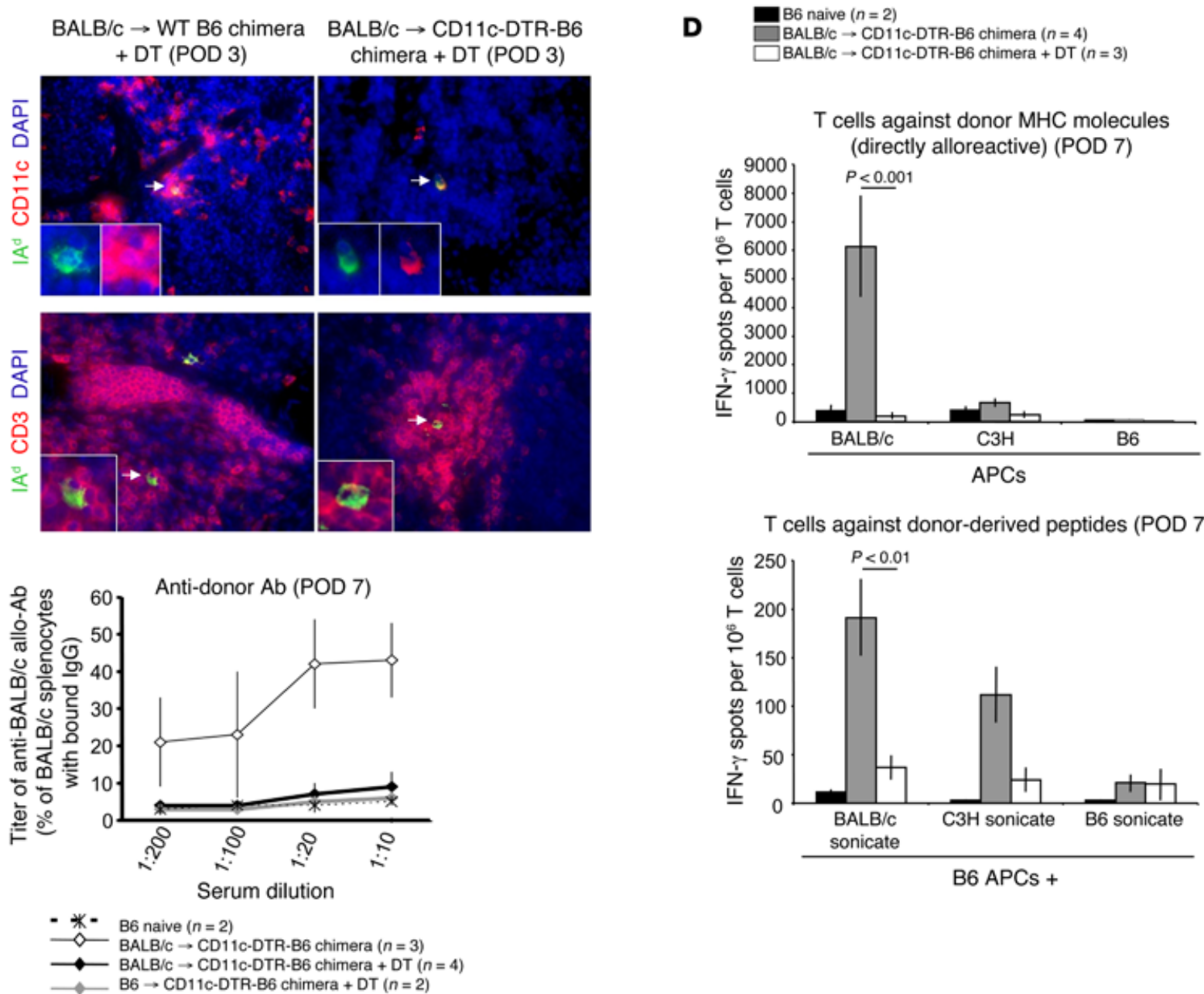

T cells against donor-derived peptides (POD 7)

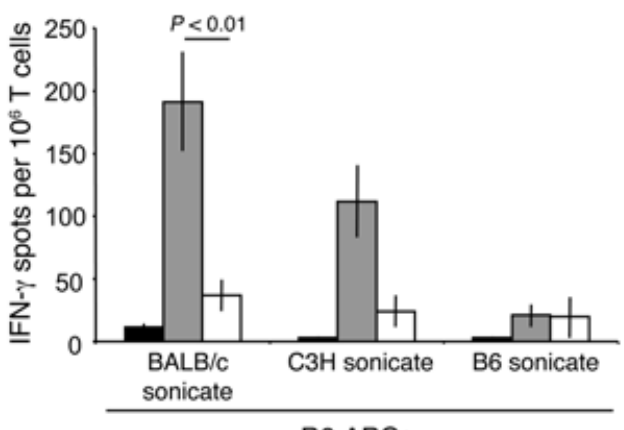

B6 APCs +

Figure 10. Recipient CDCs present donor MHC molecules to directly alloreactive T cells after heart transplantation. (A) Survival of BALB/c cardiac grafts in CD11c-DTR-B6 BM chimeras depleted of recipient cDCs. Recipient numbers are in parentheses. (B) Quantification by immunofluorescence microscopy of donor $(B A L B / c) c D C s\left(C D 11 c^{+} I A^{d+}\right)$ on tissue sections of spleens of $B 6\left(H 2^{b}\right)$ recipients, on successive PODs. Results represent the analysis of 10 panoramic sections of each spleen per POD and animal group. Results were analyzed with 1-way ANOVA followed by Tukey-Kramer multiple comparisons test. Cells were counted with MetaMorph Offline 7.7.50 software. NS, not significant; ND, not detected. (C) Top: Donor (BALB/c) cDCs detected on tissue sections of spleens from DT-treated WT B6 BM chimeras (control) and DT-injected CD11c-DTR BM chimeras were identified by expression of IA hi (green) and CD11c (red). Bottom: Homing of donor (BALB/C, IA ${ }^{\mathrm{d}+}$ CDCs (green) to splenic T cell areas (red) in DT-treated WT B6 BM chimeras (control) and DT-injected CD11cDTR BM chimeras. Arrows indicate the donor DCs shown in detail in the insets. Nuclei were stained blue with DAPI. Immunofluorescence microscopy, original magnification, $\times 400$. Sections are representative of 3 animals per variable. (D) Enzyme-linked ImmunoSpot (ELISPOT) analysis of the recipient $\mathrm{T}$ cell response against donor MHC molecules (direct pathway) or donor-derived peptides presented by recipient MHC molecules (indirect pathway) in the spleen on POD 7. Results were pooled from 3-4 mice per group. $P$ values were generated by 1-way ANOVA followed by Tukey-Kramer multiple comparisons test. (E) Anti-donor (BALB/C) Ab titers in serum on POD 7. Recipient numbers are in parentheses. 
A
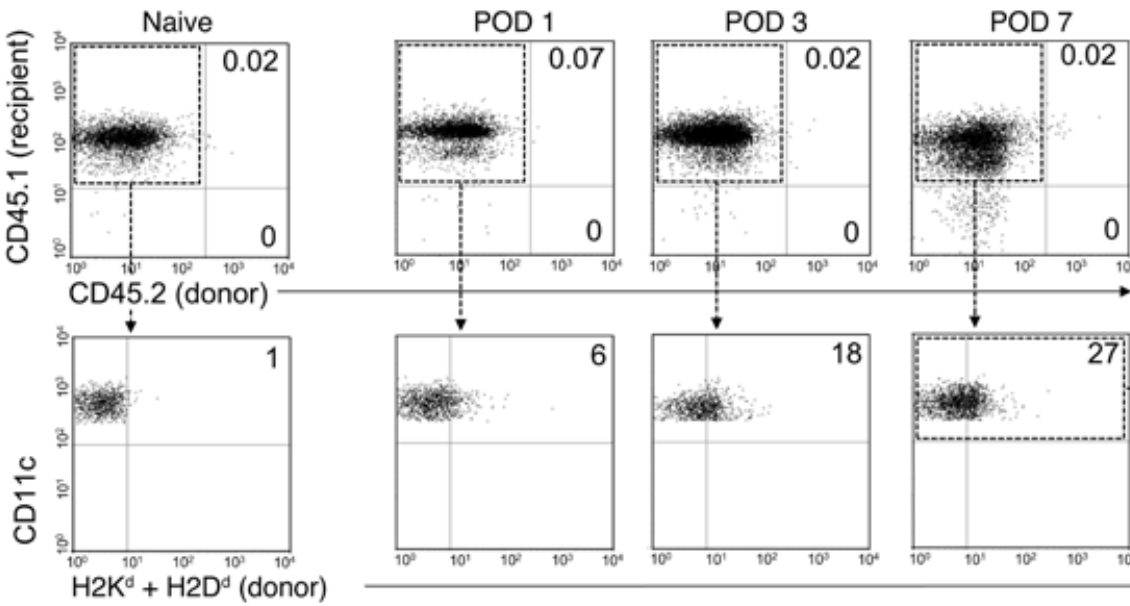

$\mathbf{B}$
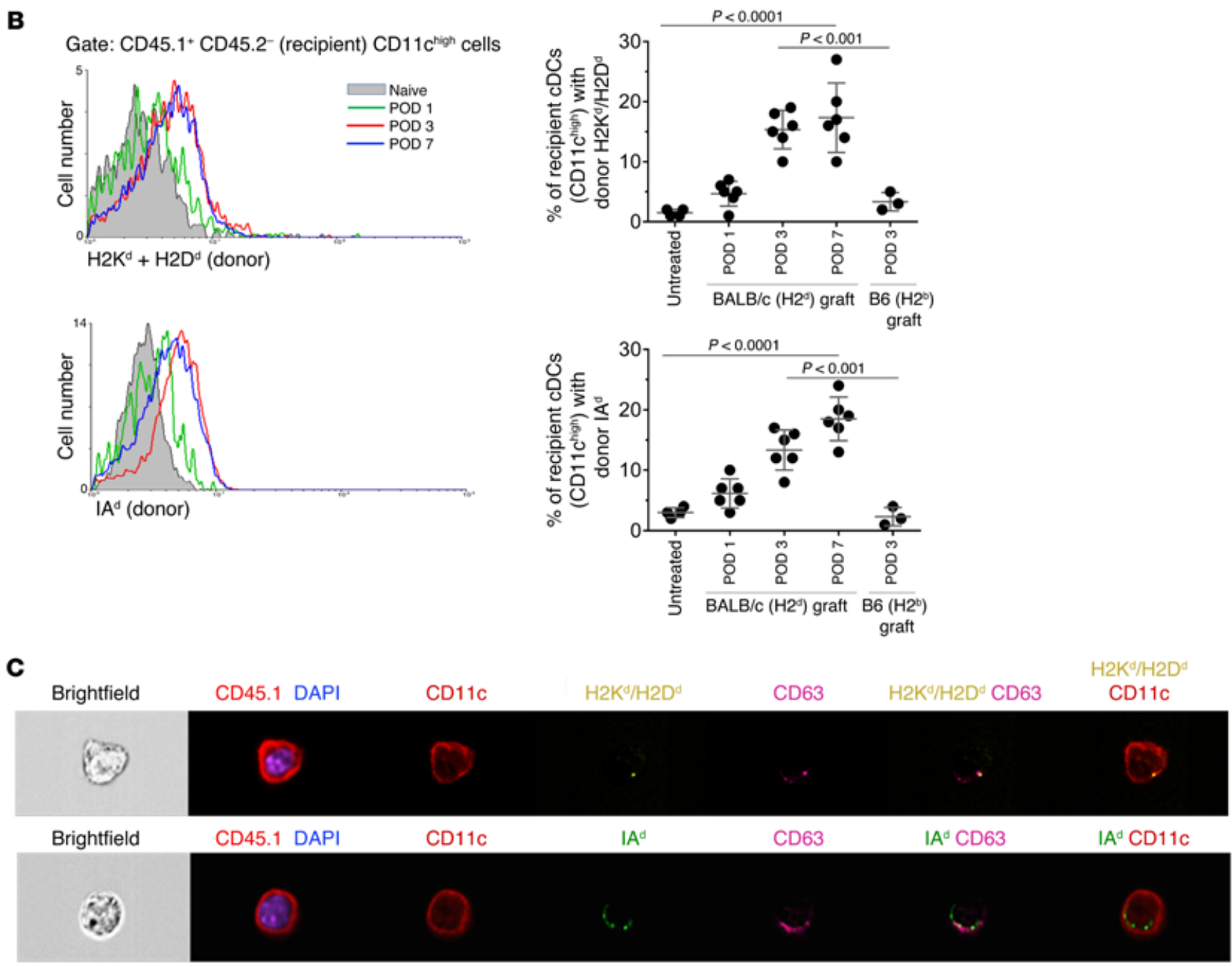

Figure 11. Transfer of donor MHC molecules after skin transplantation. (A) Top dot plots: Detection by FACS of donor (CD45.2 $2^{+}$CD45.1-) cells migrated from BALB/c (CD45.2+) fully mismatched skin allografts in B6 (CD45.1+) mice. Donor cells were undetectable by FACS within the "live cell gate" on PODs 1 , 3 , and 7, in the spleen (A) and in the draining lymph nodes (axillary + inguinal, not shown). Bottom dot plots: Detection by FACS of donor MHC class I (H2K ${ }^{d}$ $\left.+{ }^{H} 2 D^{d}\right)$ molecules on recipient (CD45.1 ${ }^{+}$CD45.2-) splenic CDCs. Recipient splenic CD8 $\alpha^{+}$and CD8 $\alpha^{-}$CDCs acquired donor MHC class I molecules. Numbers in dot plots indicate percentages of cells in the corresponding quadrants. Results are representative of 6 mice per time point. (B) Left: FACS analysis of

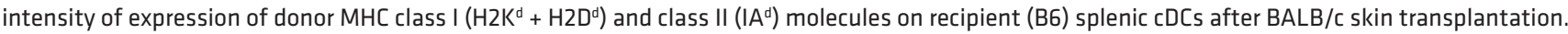
Right: Percentages of recipient (B6) splenic cDCs cross-dressed with donor MHC class I (H2K $+\mathrm{H}^{\mathrm{d}} \mathrm{D}^{\mathrm{d}}$ ) and class II (IA $)$ molecules, analyzed by FACS after $\mathrm{BALB} / \mathrm{c}$ skin transplantation. Results were pooled from 2 experiments, each with 3 mice per time point. $P$ values were generated by 1-way ANOVA followed by Tukey-Kramer multiple comparisons test. (C) Analysis by ImageStream technology of recipient (B6, CD45.1+) splenic CDCs (CD11c ${ }^{+}$) cross-dressed with donor (BALB/C) MHC class I (H2 $\left.\mathrm{K}^{\mathrm{d}}+\mathrm{H}_{2} \mathrm{D}^{\mathrm{d}}\right)$ and class II (IA $)$ molecules, both located in spots containing the exosome marker CD63. ImageStream, original magnification, $\times 60,5,000$ cells analyzed. 
DCs after BM transplantation (34-36). By the semidirect pathway, an individual DC from the recipient is able to present both (a) donor intact MHC molecules to trigger activation of directly alloreactive naive CD8 T cells and (b) donor-derived peptides in recipient (self) MHC molecules to stimulate indirectly alloreactive CD4 T cells required to provide linked help to the APC for stimulation of the naive CD8 T cells (37-39). The semidirect pathway explains how indirect-pathway $\mathrm{T}$ cells not only stimulate but also cross-regulate the function of direct-pathway $\mathrm{T}$ cells through the same recipient APC $(40,41)$. The results presented here unveil at the ultrastructural level and in vivo the mechanism of transfer of MHC molecules between donor and recipient cDCs, which is the basis of the semidirect pathway. Furthermore, they demonstrate that spreading of clusters of exosomes bearing donor MHC molecules plus APC-activating signals increases the probability that the relatively few donor DCs migrated from mouse cardiac allografts will prime effectively directly alloreactive $\mathrm{T}$ cells at the extremely low $\mathrm{DC} / \mathrm{T}$ cell ratios detected in the graft-draining lymphoid tissues.

Interestingly, until now the mechanism(s) behind passage of MHC between leukocytes in vivo has not been fully elucidated. Exchange (or unidirectional passage) of fragments of plasma membrane between donor and acceptor cells has been proposed (42). However, transfer or exchange of patches of plasma membrane between leukocytes has not been confirmed at the ultrastructural level. A more plausible alternative is that leukocytes transfer MHC through EVs, which differ in biogenesis, size, and molecular composition. MVs and apoptotic cell blebs range between 0.2 and $1 \mu \mathrm{m}$ in size and are shed from the surface membrane of living and dying cells, respectively (16). In contrast, exosomes are smaller vesicles $(30-120 \mathrm{~nm})$ of endocytic origin generated in late endosomes/MVBs, and secreted when MVBs fuse with the plasma membrane (16).

An interesting finding in our model is that transfer of MHC between cDCs was minimal when cells were separated with 0.4- $\mu \mathrm{m}$-pore transwells. In general, the conclusion of such a result is that MHC transfer occurs through capture of plasma membrane patches from donor cells, instead of transfer of free floating EVs. However, our results by EM showed that the donor MHC molecules were transferred via clusters of clumped small EVs that, because of their size and Brownian motion, were unable to pass through the $0.4-\mu \mathrm{m}$ pores. Besides, transfer of such EV clusters may also require cell-to-cell contact.

The composition of EVs depends on the lineage and activation of the parent cell. EVs released by mature DCs are enriched in $\mathrm{MHC}$, adhesion, and $\mathrm{T}$ cell costimulatory molecules $(28,43)$. Numerous studies have shown that APCs acquire MHC molecules by binding APC-derived exosomes added as free EVs to the culture medium $(28,29,43,44)$. These results have led to the idea that APCs always capture exosomes and other EVs as free-floating individual vesicles through mechanisms that do not require intercellular contact. In contrast, our work demonstrates that donor migrating DCs transfer MHC molecules by passing clusters of small EVs - instead of individual EVs - to recipient cDCs. Release of small EVs to the confined area of the intercellular space limits dispersion of the EVs by Brownian motion, allowing retention of the transferred EVs within a confined area of the acceptor
cDC surface. EVs carry on the surface adhesion molecules and their ligands that could stabilize the EV cluster.

Transfer of RFP-tagged exosomes from BALB/c migrating BMDCs to lymph node- or spleen-resident $\mathrm{B} 6 \mathrm{YFP}^{+} \mathrm{cDCs}$ occurred within minutes. We also demonstrated that the transferred exosomes were retained on the acceptor cDC for hours, or internalized. Transfer of clusters of exosomes - and potentially other types of donor-derived EVs - likely facilitates organization of the immunological synapse (much bigger in diameter than an individual exosome), and provides sufficient density of donor MHC molecules to promote activation of directly alloreactive naive $\mathrm{T}$ cells.

Unlike other types of EVs, exosomes released by activated DCs promoted activation of acceptor cDCs in our model, which depended on the dose of exosomes transferred and the preservation of the vesicular structure. Previous studies have shown that exosomes released by mast cells (45), red blood cells (46), or pathogen-infected macrophages (47) promote activation of DCs and monocytes. Interestingly, exosomes released by mature DCs are enriched in miR-155 and contain heat-shock proteins, both inducers of DC activation $(48,49)$.

If donor migrating $\mathrm{cDCs}$ transfer through EVs donor $\mathrm{MHC}$ molecules and APC-activating signals to recipient cDCs, depletion of the latter cells should interfere with priming of directly alloreactive T cells. Accordingly, in the absence of recipient cDCs, $\mathrm{T}$ cell priming against donor MHC molecules was drastically impaired, and therefore cardiac allograft rejection was delayed. This confirms that in our model (nonsensitized recipients), the relatively few donor DCs homed in the spleen were not sufficient to prime directly alloreactive naive T cells. These donor DCs rather function as antigen-transporting cells, spreading donor MHC and APC-activating signals to a higher number of recipient cDCs in lymphoid tissues.

Certain microbial components, allergens, and model or tumor antigens are sorted into exosomes released by APCs, including DCs (15). Although our findings are in transplantation, transfer of preformed self MHC:peptide complexes via exosomes or other EVs from migrating DCs to a higher number of lymphoid tissue-resident $\mathrm{cDCs}$ could also enhance $\mathrm{T}$ cell stimulation during microbial infections, allergies, or vaccinations. In this regard, Smyth et al. have elegantly shown that virally infected migrating cDCs transfer functional MHC class I:peptide complexes to splenic $\mathrm{cDCs}$, which then primed CD8 T cells against virus-encoded antigens $(30,50)$. Cross-dressed DCs are also relevant for expansion of memory CD8 T cells after viral infections (29). DCs designed for vaccination transfer in vitro MHC class I:peptides via exosomes to other DCs for efficient CD8 T cell priming (51). Thus, amplification of the $\mathrm{T}$ cell response via passage of exosomes between migrating DCs and cDCs of lymphoid tissues is likely a generalized phenomenon not restricted to transplant immunity.

In summary, our study has identified dissemination within graft-draining lymph nodes of donor-derived exosomes bearing MHC molecules and APC-activating signals, as a key component of the effectiveness of the response against non-self MHC molecules in organ transplantation. We demonstrated that this is a general phenomenon that occurs in vascularized (heart) and nonvascularized (skin) transplant models. Our findings do not exclude the potential contribution of other types of donor-derived EVs in 
T and B allosensitization, or other subsets of recipient APCs. This phenomenon may not be restricted to secondary lymphoid tissues but could also be operational in the graft where recipient APCs are cross-dressed and present donor MHC to recipient T cells (52). These recipient APCs could activate memory T cells that infiltrate the graft - bypassing lymphoid tissues - or could return to the secondary lymphoid tissues to prime T cells.

\section{Methods}

Mice and reagents. Male C57BL/6 (B6), BALB/c, CB6F1/J, C3H/HeJ

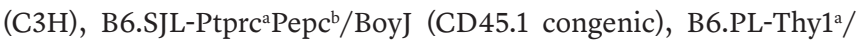
CyJ, B6.Cg-Tg(Itgax-venus)1Mnz/J (CD11c-YFP), and B6.FVB$\mathrm{Tg}$ (Itgax-DTR/eGFP) ${ }_{57} \mathrm{Lan} / \mathrm{J}$ (CD11c-DTR) mice were purchased from The Jackson Laboratory. 2C and 1H3.1 RAG1 ${ }^{\mathrm{KO}}$ B6 mice were bred in our animal facility. Cytokines and Abs were purchased from PeproTech, and Abs from Abcam, BD Pharmingen, eBioscience, Invitrogen-Molecular Probes, Jackson ImmunoResearch Laboratories, Thermo Scientific, Santa Cruz Biotechnology, or Cedarlane. DT was purchased from Sigma-Aldrich.

Heart and skin transplantation. Heterotopic (abdomen) vascularized cardiac transplantation was performed with the method of Corry et al. (53). Palpation for heartbeat was conducted daily to determine organ survival. For the skin transplants, ears were removed from the donors and then split with forceps into dorsal and ventral halves. The dorsal halves, which contain no cartilage, were transplanted on beds prepared on the left and right lateral flanks of the recipient mice.

Estimation of donor cell number by quantitative PCR. DNA was extracted with TRIzol (Invitrogen) from spleens and mediastinal and retroperitoneal lymph nodes of B6 mice untreated or 1, 2, 3, or 7 days after they received BALB/c heart grafts. DNA was diluted in DNase/ RNase-free water (100 $\mathrm{ng} / \mu \mathrm{l})$, and BALB/c DNA was amplified and quantified by quantitative PCR with a StepOne thermal cycler (Applied Biosystems), using primers targeted to a region of the MHC class II IE $\alpha$ gene (H2-E $\alpha$ ) encoded in the BALB/c H2 ${ }^{\mathrm{d}}$ haplotype (GenBank K00971) and absent in the B6 genome. Primers were designed with the Invitrogen custom primer software Oligo Perfect Designer. H2-E $\alpha$ forward 5'-AGAAAATGGCCACAATTGGA-3' and reverse 5'-TCTGGGAGCTCATCAGAACA-3' primers were from Invitrogen. DNA integrity and loading were normalized using the housekeeping gene S15 (forward primer 5'-GTGGAAGCTGGTGGATTCAT-3' and reverse primer $5^{\prime}$-ACACCCAGAAAGGAACATGC-3'). Reactions were done in $50 \mu \mathrm{l}$ of total volume with $1.2 \mu \mathrm{g}$ of genomic DNA, which is equivalent to approximately $10^{6}$ cells. Final concentration $(200 \mathrm{mM})$ of each primer was mixed with $25 \mu$ l of $2 \times$ Fast SYBR Green Master Mix (Applied Biosystems). DNase- and RNase-free water was used to bring the reactions to a final volume of $50 \mu$ l. The quantitative PCR cycling conditions were as follows: initial hold for 20 seconds at $95^{\circ} \mathrm{C}$, followed by 40 cycles of $95^{\circ} \mathrm{C}$ for 15 seconds (denaturing), and $60^{\circ} \mathrm{C}$ for 1 minute (annealing/extending). For standardization, BALB/c DNA was serially diluted into $\mathrm{B} 6 \mathrm{DNA}$, resulting in $\mathrm{BALB} / \mathrm{c} / \mathrm{B} 6 \mathrm{DNA}$ ratios from 1:10 to 1:10 ${ }^{6}$. DNase/RNase-free water and DNA extracted from untreated naive B6 mice were used as controls. Normalization to control for DNA integrity and loading, and to compensate for inter-PCR variation, was performed with the Pfaffl method. The PCR-derived standard curve had a sensitivity of $1 \mathrm{BALB} / \mathrm{c}$ cell per $10^{6} \mathrm{~B} 6$ splenocytes.

Assay of MHC transfer between DCs. Splenic DCs were isolated from $\mathrm{B} 6$ or $\mathrm{BALB} / \mathrm{c}$ mice injected (s.c.) with $10^{7}$ to $2 \times 10^{7} \mathrm{~B} 16$ mela- noma cells stably transfected with the human Flt-3 ligand gene (A. Fierro, University of Chile, Santiago, Chile). After 14 days, spleens were removed and digested with collagenase, and DC-enriched suspensions were obtained by centrifuging of the splenocytes over $16 \%$ Histodenz gradients. cDCs were then purified with CD11c magnetic beads (Miltenyi Biotec) (DC purity $\geq 95 \%$ ). BALB/c cDCs were labeled with $7.5 \mu \mathrm{M}$ Vybrant CFDA SE Cell Tracer. Unlabeled B6 cDCs were cocultured with CFSE-labeled BALB/c cDCs, at 1:1 ratio, in 24-well plates $(500,000 \mathrm{cDCs}$ of each per well), in $1 \mathrm{ml}$ of complete medium supplemented with $1,000 \mathrm{U} / \mathrm{ml}$ of GM-CSF, alone or with increasing concentrations of imipramine (Sigma-Aldrich) or the peptide Z-DEVD-FMK (MBL). Unlabeled B6 cDCs were also cocultured at 1:1 cell ratio, with CFSE-labeled BALB/c BMDCs previously transfected with Rab27a siRNAs or control (eGFP) siRNAs. For assessment of MHC transfer efficiency, a fixed number of B6 cDCs (500,000 cells per well) was cocultured with 2-fold serial dilutions of CFSE-labeled $\mathrm{BALB} / \mathrm{c}$ cDCs. In some experiments, the CFSE-labeled BALB/c cDCs were cultured separated from the unlabeled B $6 \mathrm{cDCs}$ by $0.4-\mu \mathrm{m}$-pore transwells (Corning). After 20 hours of culture, cells were harvested and CDC clusters disaggregated by rinsing with ice-cold 0.01-M EDTA in PBS and pipetting. Dead cells were removed by centrifugation over a Lympholyte $\mathrm{M}$ gradient (20 minutes, $4^{\circ} \mathrm{C}, 1,500 \mathrm{~g}$ ) (Cedarlane). cDCs were labeled with PE-H2K ${ }^{\mathrm{d}}, \mathrm{APC}^{-\mathrm{IA}^{\mathrm{d}}}$, and PerCP-Cy5.5-CD11c Abs, fixed in PFA, and analyzed by FACS.

Confocal microscopy. BALB/c DCs were generated from BM precursor cells cultured in RPMI 1640 supplemented with 10\% FCS, GM-CSF $(1,000 \mathrm{U} / \mathrm{ml})$, and IL-4 (500 U/ml). Day 6 BMDCs were purified with CD11c magnetic beads and transduced $(\mathrm{MOI}=100)$ with the recombinant adenovirus (RAd)-CD63-RFP encoding RFP cDNA (Evrogen) fused to mouse CD63 cDNA (Sino Biological Inc.), or with the control vector RAd-RFP (encoding RFP cDNA alone) or RAd-Empty (no transgene). One hour after transduction, BMDCs were labeled with Hoechst stain (Invitrogen), washed 3 times, and injected s.c. (footpad, $2 \times 10^{6}$ BMDCs in $50 \mu \mathrm{l} \mathrm{PBS}$ ) or i.v. (tail vein, $5 \times 10^{6}$ BMDCs in $200 \mu \mathrm{l} \mathrm{PBS}$ ) in CD11c-YFP B6 mice. Before injection, CD11c ${ }^{+}$BMDC purity was $\geq 95 \%$ and cell viability $\geq 98 \%$ (by FACS). After BMDC injection (16 hours), the ipsilateral popliteal lymph nodes or the spleens were harvested. The spleens were cut into $400-\mu \mathrm{m}$-thick explants with a vibratome. The lymph nodes and spleen explants were embedded in $3 \%$ low-melting-temperature agarose and mounted in a custom-designed closed chamber perfused with medium bubbled with $95 \% \mathrm{O}_{2} / 5 \% \mathrm{CO}_{2}$, at $37^{\circ} \mathrm{C}$. Samples were imaged with a Nikon Eclipse Ti confocal system or with a Nikon $\mathrm{AlMP}^{+}$multiphoton confocal microscope. Time-lapse (3 minutes) 3D series of up to 5 different locations of each sample were collected up to a 4 -hour period. Images were analyzed with the imaging software NIS-Elements version 4.20 (Advanced Research, Nikon) and Imaris X64 version 8 (Bitplane, Oxford Instruments).

Purification of EVs. MVs and exosomes were purified from culture supernatants of BALB/c BMDCs maintained in medium with exosome-free FCS during the last 48 hours of culture. To promote DC maturation, in some experiments BALB/c BMDCs were incubated with IL-1 $\beta(20 \mathrm{ng} / \mathrm{ml})$ and TNF- $\alpha(50 \mathrm{ng} / \mathrm{ml})$ during the last 24 hours of culture. For isolation of MVs, BMDC (day 6) culture supernatants were centrifuged at $300 \mathrm{~g}$ (10 minutes), 1,200 $\mathrm{g}$ (20 minutes), and $10,000 \mathrm{~g}$ (30 minutes), all at $4^{\circ} \mathrm{C}$. The $10,000-g$ pellets were collected, washed in PBS, and centrifuged at 10,000 $g$ (30 minutes), and the final pellets were harvested in $200 \mu$ l of PBS. 
For isolation of highly purified exosomes, BALB/c BMDC (day 6) culture supernatants were centrifuged at $300 \mathrm{~g}$ (10 minutes), $1,200 \mathrm{~g}$ (20 minutes), and 10,000 $g$ (30 minutes) and the supernatants ultrafiltered $(2,000 \mathrm{~g}, 20$ minutes) through Vivacell 100 filters. The filtered supernatants were adjusted to $11 \mathrm{ml}$ with PBS and centrifuged at $100,000 \mathrm{~g}$ for 90 minutes. The 100,000- $g$ pellets with the exosomes were floated on a continuous sucrose gradient $(0.25-2.5 \mathrm{M})$ as previously described (54) with minor modifications. The fractions containing the exosomes were pooled, adjusted to $11 \mathrm{ml}$ with PBS, and centrifuged at 100,000 $\mathrm{g}$ for 90 minutes, and the pellets were collected in $200 \mu \mathrm{l}$ of PBS.

The amount of protein in the EV preparations was assessed with a NanoDrop 2000c, the morphology of the EVs was analyzed by EM, and the average size of the EVs was measured with an LM10 NanoSight instrument equipped with a high-sensitivity EMCCD camera and NTA 2.0 software (NanoSight) (55). To test the effect of EVs on DC maturation, day 5 B6 BMDCs (200,000 cells per well of 48-well plates) were left untreated or incubated with different concentrations of MVs or exosomes (generated from immature or mature BALB/c BMDCs) in $1 \mathrm{ml}$ of complete medium supplemented with GM-CSF $(1,000 \mu \mathrm{m} / \mathrm{ml})$, and $10 \%$ exosome-free FCS. After 16 hours, the B6 BMDCs were labeled with fluorochrome Abs against B6 MHC class II (IA $\left.{ }^{\mathrm{b}}\right), \mathrm{CD} 40, \mathrm{CD} 80, \mathrm{CD} 86$, or PD-L1, and analyzed by flow cytometry. Alternatively, the B6 BMDCs were used as stimulators of CFSElabeled $\mathrm{C} 3 \mathrm{H}$ naive $\mathrm{T}$ cells in mixed leukocyte cultures. After 4 days, cells were harvested and labeled with fluorochrome Abs against CD4, CD8, and CD11c, and the extent of CFSE dilution in the T cells was analyzed by flow cytometry.

Preparation of irradiation BM chimeras. Male WT B6 mice (5-6 weeks old) were $\gamma$-irradiated with 550 rad twice, 6 hours apart, and 2 hours later were injected i.v. with $10^{7} \mathrm{BM}$ cells from CD11c-DTR-B6 mice or from WT B6 mice, the latter for control WT B6 BM chimeras. Animals were kept in autoclaved cages, provided with sterile water with Sulfatrim during the first week, and used as recipients 8 weeks after BM infusion.
Statistics. GraphPad Prism was used for statistical analyses. Results are expressed as mean \pm SD. Multiple comparisons on a single data set were done by 1-way ANOVA, followed by Tukey-Kramer multiple comparisons test. Graft survival was compared by Kaplan-Meier analysis and the log-rank test. In all experiments, a $P$ value less than 0.05 was considered significant.

Study approval. Studies were approved by the IACUC of the University of Pittsburgh.

Supplemental material. The supplemental material (online) includes 12 Supplemental Figures, 8 Supplemental Videos, and Supplemental Methods.

\section{Author contributions}

QL, DMR-C, SJD, WJS, ATL, and AEM performed experiments. DBS and MLGS conducted EM analysis. GE designed and cloned the recombinant adenovirus. GAG and SCW performed highresolution confocal microscopy. QL, ATL, and AEM designed the research and wrote the paper.

\section{Acknowledgments}

We thank L.D. Falo for providing the B6 CD11c-DTR-eGFP mice, G. Chalasani for the $2 \mathrm{C}$ mice, and A. Gamboto and Kaori Okada for help in the generation of viral vectors. This work was supported by grants from the American Heart Association (14GRNT19810000 to A.E. Morelli), the NIH (R01-HL130191 to A.E. Morelli, R01AR068249 to A.T. Larregina, and F30-DK082131 to S.J. Divito), and the National Natural Science Foundation of China (NSFC81401318 to Q. Liu), and funds from the T.E. Starzl Transplantation Institute (to A.E. Morelli).

Address correspondence to: Adrian E. Morelli, W1556 Biomedical Science Tower, 200 Lothrop Street, Pittsburgh, Pennsylvania 15261, USA. Phone: 412.624.2193; E-mail: morelli@pitt.edu.
1. Lakkis FG, Arakelov A, Konieczny BT, Inoue Y. Immunologic 'ignorance' of vascularized organ transplants in the absence of secondary lymphoid tissue. Nat Med. 2000;6(6):686-688.

2. Larsen CP, Morris PJ, Austyn JM. Migration of dendritic leukocytes from cardiac allografts into host spleens. A novel pathway for initiation of rejection. J Exp Med. 1990;171(1):307-314.

3. Chen Y, Demir Y, Valujskikh A, Heeger PS. The male minor transplantation antigen preferentially activates recipient $\mathrm{CD} 4^{+} \mathrm{T}$ cells through the indirect presentation pathway in vivo. J Immunol. 2003;171(12):6510-6518.

4. Brennan TV, et al. Preferential priming of alloreactive T cells with indirect reactivity. Am J Transplant. 2009;9(4):709-718.

5. Yamada A, Chandraker A, Laufer TM, Gerth AJ, Sayegh MH, Auchincloss H. Recipient MHC class II expression is required to achieve long-term survival of murine cardiac allografts after costimulatory blockade. JImmunol. 2001;167(10):5522-5526.

6. Zheng XX, et al. The role of donor and recipient B7-1 (CD80) in allograft rejection. J Immunol. 1997;159(3):1169-1173.

7. Mandelbrot DA, et al. Expression of B7 mol- ecules in recipient, not donor, mice determines the survival of cardiac allografts. J Immunol. 1999;163(7):3753-3757.

8. Yu G, Xu X, Vu MD, Kilpatrick ED, Li XC. NK cells promote transplant tolerance by killing donor antigen-presenting cells. J Exp Med. 2006;203(8):1851-1858.

9. Laffont S, Seillet C, Ortaldo J, Coudert JD, Guéry JC. Natural killer cells recruited into lymph nodes inhibit alloreactive T-cell activation through perforin-mediated killing of donor allogeneic dendritic cells. Blood. 2008;112(3):661-671.

10. Laffont $\mathrm{S}$, et al. CD8 ${ }^{+} \mathrm{T}$-cell-mediated killing of donor dendritic cells prevents alloreactive T helper type-2 responses in vivo. Blood. 2006;108(7):2257-2264.

11. Garrod KR, Liu FC, Forrest LE, Parker I, Kang SM Cahalan MD. NK cell patrolling and elimination of donor-derived dendritic cells favor indirect alloreactivity. JImmunol. 2010;184(5):2329-2336.

12. Divito SJ, et al. Endogenous dendritic cells mediate the effects of intravenously injected therapeutic immunosuppressive dendritic cells in transplantation. Blood. 2010;116(15):2694-2705.

13. Théry C, Ostrowski M, Segura E. Membrane vesicles as conveyors of immune responses. Nat
Rev Immunol. 2009;9(8):581-593.

14. Raposo G, Stoorvogel W. Extracellular vesicles: exosomes, microvesicles, and friends. JCell Biol. 2013;200(4):373-383.

15. Robbins PD, Morelli AE. Regulation of immune responses by extracellular vesicles. Nat Rev Immunol. 2014;14(3):195-208.

16. Colombo M, Raposo G, Théry C. Biogenesis, secretion, and intercellular interactions of exosomes and other extracellular vesicles. Annu Rev Cell Dev Biol. 2014;30:255-289.

17. Fleissner F, Goerzig Y, Haverich A, Thum T. Microvesicles as novel biomarkers and therapeutic targets in transplantation medicine. Am J Transplant. 2012;12(2):289-297.

18. Tkach M, Théry C. Communication by extracellular vesicles: where we are and where we need to go. Cell. 2016;164(6):1226-1232.

19. Ostrowski M, et al. Rab27a and Rab27b control different steps of the exosome secretion pathway. Nat Cell Biol. 2010;12(1):19-30.

20. Bianco F, et al. Acid sphingomyelinase activity triggers microparticle release from glial cells. EMBO J. 2009;28(8):1043-1054.

21. Zhang J, Reedy MC, Hannun YA, Obeid LM. Inhibition of caspases inhibits the release of 
apoptotic bodies: Bcl-2 inhibits the initiation of formation of apoptotic bodies in chemotherapeutic agent-induced apoptosis. J Cell Biol. 1999;145(1):99-108.

22. Li J, et al. Exosomes mediate the cell-to-cell transmission of IFN- $\alpha$-induced antiviral activity. Nat Immunol. 2013;14(8):793-803.

23. Celli S, Albert ML, Bousso P. Visualizing the innate and adaptive immune responses underlying allograft rejection by two-photon microscopy. Nat Med. 2011;17(6):744-749.

24. Steptoe RJ, Patel RK, Subbotin VM, Thomson AW. Comparative analysis of dendritic cell density and total number in commonly transplanted organs: morphometric estimation in normal mice. Transpl Immunol. 2000;8(1):49-56.

25. Emerson SG, Cone RE. I-Kk and H-2Kk antigens are shed as supramolecular particles in association with membrane lipids. J Immunol. 1981;127(2):482-486.

26. Sachs DH, Kiszkiss P, Kim KJ. Release of Ia antigens by a cultured B cell line. J Immunol. 1980;124(5):2130-2136.

27. Raposo G, et al. B lymphocytes secrete antigen-presenting vesicles. JExp Med.1996;183(3):1161-1172.

28. Montecalvo A, et al. Exosomes as a short-range mechanism to spread alloantigen between dendritic cells during $\mathrm{T}$ cell allorecognition. J Immunol. 2008;180(5):3081-3090.

29. Wakim LM, Bevan MJ. Cross-dressed dendritic cells drive memory $\mathrm{CD} 8^{+} \mathrm{T}$-cell activation after viral infection. Nature. 2011;471(7340):629-632.

30. Smyth LA, et al. Acquisition of MHC:peptide complexes by dendritic cells contributes to the generation of antiviral $\mathrm{CD} 8^{+} \mathrm{T}$ cell immunity in vivo. JImmunol. 2012;189(5):2274-2282.

31. Zhang QJ, et al. Trogocytosis of MHC-I/peptide complexes derived from tumors and infected cells enhances dendritic cell cross-priming and promotes adaptive T cell responses. PLoS One. 2008;3(8):e3097.

32. Harshyne LA, Watkins SC, Gambotto A, BarrattBoyes SM. Dendritic cells acquire antigens from live cells for cross-presentation to CTL. J Immunol. 2001;166(6):3717-3723.

33. Herrera OB, et al. A novel pathway of alloantigen presentation by dendritic cells. JImmunol. 2004;173(8):4828-4837.
34. Brown K, Sacks SH, Wong W. Extensive and bidirectional transfer of major histocompatibility complex class II molecules between donor and recipient cells in vivo following solid organ transplantation. FASEB J. 2008;22(11):3776-3784.

35. Brown K, Sacks SH, Wong W. Coexpression of donor peptide/recipient MHC complex and intact donor MHC: evidence for a link between the direct and indirect pathways. Am J Transplant. 2011;11(4):826-831.

36. Markey KA, et al. Cross-dressing by donor dendritic cells after allogeneic bone marrow transplantation contributes to formation of the immunological synapse and maximizes responses to indirectly presented antigen. J Immunol. 2014;192(11):5426-5433.

37. Jiang S, Herrera O, Lechler RI. New spectrum of allorecognition pathways: implications for graft rejection and transplantation tolerance. Curr Opin Immunol. 2004;16(5):550-557.

38. Smyth LA, Afzali B, Tsang J, Lombardi G, Lechler RI. Intercellular transfer of MHC and immunological molecules: molecular mechanisms and biological significance. Am J Transplant. 2007;7(6):1442-1449.

39. Sivaganesh S, et al. Copresentation of intact and processed MHC alloantigen by recipient dendritic cells enables delivery of linked help to alloreactive CD8 T cells by indirect-pathway CD4 T cells. JImmunol. 2013;190(11):5829-5838.

40. Lee RS, Grusby MJ, Glimcher LH, Winn HJ, Auchincloss H. Indirect recognition by helper cells can induce donor-specific cytotoxic T lymphocytes in vivo. J Exp Med. 1994;179(3):865-872.

41. Wise MP, Bemelman F, Cobbold SP, Waldmann H. Linked suppression of skin graft rejection can operate through indirect recognition. J Immunol. 1998;161(11):5813-5816.

42. Caumartin J, Lemaoult J, Carosella ED. Intercellular exchanges of membrane patches (trogocytosis) highlight the next level of immune plasticity. Transpl Immunol. 2006;17(1):20-22.

43. Segura $\mathrm{E}$, et al. ICAM-1 on exosomes from mature dendritic cells is critical for efficient naive T-cell priming. Blood. 2005;106(1):216-223.

44. Théry C, Duban L, Segura E, Véron P, Lantz O, Amigorena S. Indirect activation of naive $\mathrm{CD} 4^{+}$ $\mathrm{T}$ cells by dendritic cell-derived exosomes. $\mathrm{Nat}$
Immunol. 2002;3(12):1156-1162.

45. Skokos D, et al. Mast cell-derived exosomes induce phenotypic and functional maturation of dendritic cells and elicit specific immune responses in vivo. JImmunol. 2003;170(6):3037-3045.

46. Danesh A, et al. Exosomes from red blood cell units bind to monocytes and induce proinflammatory cytokines, boosting T-cell responses in vitro. Blood. 2014;123(5):687-696.

47. Bhatnagar S, Shinagawa K, Castellino FJ, Schorey JS. Exosomes released from macrophages infected with intracellular pathogens stimulate a proinflammatory response in vitro and in vivo. Blood. 2007;110(9):3234-3244.

48. Montecalvo A, et al. Mechanism of transfer of functional microRNAs between mouse dendritic cells via exosomes. Blood. 2012;119(3):756-766.

49. Théry C, et al. Molecular characterization of dendritic cell-derived exosomes. Selective accumulation of the heat shock protein hsc73. J Cell Biol. 1999;147(3):599-610.

50. Smyth LA, et al. The relative efficiency of acquisition of MHC:peptide complexes and crosspresentation depends on dendritic cell type. JImmunol. 2008;181(5):3212-3220.

51. Andre F, et al. Exosomes as potent cell-free peptide-based vaccine. I. Dendritic cell-derived exosomes transfer functional MHC class I/ peptide complexes to dendritic cells. JImmunol. 2004;172(4):2126-2136.

52. Chalasani G, Dai Z, Konieczny BT, Baddoura FK, Lakkis FG. Recall and propagation of allospecific memory T cells independent of secondary lymphoid organs. Proc Natl Acad Sci U S A. 2002;99(9):6175-6180.

53. Corry RJ, Winn HJ, Russell PS. Primarily vascularized allografts of hearts in mice. The role of $\mathrm{H}-2 \mathrm{D}, \mathrm{H}-2 \mathrm{~K}$, and non-H-2 antigens in rejection. Transplantation. 1973;16(4):343-350.

54. Théry C, Amigorena S, Raposo G, Clayton A. Isolation and characterization of exosomes from cell culture supernatants and biological fluids. Curr Protoc Cell Biol. 2006; Chapter 3:Unit 3.22.

55. Lötvall J, et al. Minimal experimental requirements for definition of extracellular vesicles and their functions: a position statement from the International Society for Extracellular Vesicles. JExtracell Vesicles. 2014;3:26913. 